ESAIM: COCV 20 (2014) 236-268

DOI: $10.1051 / \mathrm{cocv} / 2013063$
ESAIM: Control, Optimisation and Calculus of Variations

www.esaim-cocv.org

\title{
CONTROLLABILITY OF 3D LOW REYNOLDS NUMBER SWIMMERS
}

\author{
JÉrôme LohÉAC ${ }^{1}$ And AleXandre MunnieR ${ }^{2}$
}

\begin{abstract}
In this article, we consider a swimmer (i.e. a self-deformable body) immersed in a fluid, the flow of which is governed by the stationary Stokes equations. This model is relevant for studying the locomotion of microorganisms or micro robots for which the inertia effects can be neglected. Our first main contribution is to prove that any such microswimmer has the ability to track, by performing a sequence of shape changes, any given trajectory in the fluid. We show that, in addition, this can be done by means of arbitrarily small body deformations that can be superimposed to any preassigned sequence of macro shape changes. Our second contribution is to prove that, when no macro deformations are prescribed, tracking is generically possible by means of shape changes obtained as a suitable combination of only four elementary deformations. Eventually, still considering finite dimensional deformations, we state results about the existence of optimal swimming strategies on short time intervals, for a wide class of cost functionals.
\end{abstract}

Mathematics Subject Classification. 74F10, 70S05, 76B03, 93B27.

Received February 27, 2012. Revised December 3, 2012.

Published online January 27, 2014.

\section{INTRODUCTION}

\subsection{Context}

Relevant models for the locomotion of microorganisms can be tracked back to the work of Taylor [18], Lighthill [11,12], and Childress [7]. Purcell explains in [14] that these sort of animals are of the order of a micron in size and they move around with a typical speed of 30 micron/sec. These data lead the flow regime to be characterized by a very small Reynolds number. For such swimmers, inertia effects play no role and the motion is entirely determined by the friction forces.

In this article, the swimmer is modeled as a self deforming-body. By changing its shape, it sets the surrounding fluid into motion and generates hydrodynamics forces used to propel and steer itself. We are interested in investigating whether the microswimmer is able to control its trajectory by means of appropriate shape deformations (as real microorganisms do). This question has already be tackled in some specific cases. Let us mention [16] (the authors study the motion of infinite cylinders with various cross sections and the swimming of spheres

Keywords and phrases. Locomotion, biomechanics, stokes fluid, geometric control theory.

1 Both authors are with Institut Élie Cartan UMR 7502, Université de Lorraine, CNRS, INRIA, B.P. 239, 54506

Vandoeuvre-lès-Nancy Cedex, France. jerome.loheac@univ-lorraine.fr

2 INRIA Nancy Grand Est, Projet CORIDA. Authors both supported by ANR CISIFS. Second author supported by ANR GAOS. alexandre.munnier@univ-lorraine.fr 
undergoing infinitesimal shape variations) and [2] (in which the 1D controllability of a swimmer made of three spheres is investigated). The well-posedness of the dynamics has been investigated in [13].

Our contribution to this question is several folds. First, we give a definitive answer to the control problem in the general case: the swimmer we consider has any shape at rest (obtained as the image by a $C^{1}$ diffeomorphism of the unit ball) and can undergo any kind of shape deformations (as long as they can also be obtained as images of the unit ball by $C^{1}$ diffeomorphisms). With these settings, we prove that the dynamical system governing the swimmer's motion in the fluid is controllable in the following sense: for any prescribed trajectory (i.e. given positions and orientations of the swimmer at every moment) there exists a sequence of shape changes that make it swim arbitrarily close to this trajectory. A somewhat surprising additional result is that this can be done by means of arbitrarily small shape changes which can be superimposed to any preassigned macro deformations (this is called the ability of synchronized swimming in the sequel). Second, when no macro deformations are prescribed (this is called freestyle swimming in the paper), we prove that the ability of tracking any trajectory is possible by means of shape changes obtained as an appropriate combination of only four elementary deformations (satisfying some generic assumptions). Third, we state a result about the existence of optimal swimming on short time intervals.

Notice that the paper follows the lines of [6] in which the authors study the controllability of a swimmer in a perfect fluid.

\subsection{Modeling}

\section{Kinematics}

Two frames are required in the modeling: The first one $\mathbf{E}:=\left(\mathbf{E}_{1}, \mathbf{E}_{2}, \mathbf{E}_{3}\right)$ is fixed and Galilean and the second one $\mathbf{e}:=\left(\mathbf{e}_{1}, \mathbf{e}_{2}, \mathbf{e}_{3}\right)$ is moving (see Fig. 1).

At any moment, there exist a rotation matrix $R \in \mathrm{SO}(3)$ and a vector $\mathbf{r} \in \mathbf{R}^{3}$ such that, if $X:=\left(X_{1}, X_{2}, X_{3}\right)^{*}$ and $x:=\left(x_{1}, x_{2}, x_{3}\right)^{*}$ are the coordinates of a same vector in respectively $\mathbf{E}$ and $\mathbf{e}$, then the following equality holds:

$$
X=R x+\mathbf{r}
$$

In the sequel, we will refer to the $X$-coordinates and the $x$-coordinates, depending on whether the quantities are expressed in the frame $\mathbf{E}$ or $\mathbf{e}$.

The vector $\mathbf{r}$ is meant to give the position of the swimmer and the matrix $R$ its orientation .

The rigid displacement of the swimmer, on a time interval $[0, T](T>0)$, is thoroughly described by the functions

$$
t \in[0, T] \mapsto R(t) \in \mathrm{SO}(3) \quad \text { and } \quad t \in[0, T] \mapsto \mathbf{r}(t) \in \mathbf{R}^{3},
$$

which are the unknowns of our problem. Denoting their time derivatives by $\dot{R}$ and $\dot{\mathbf{r}}$, we can define the linear velocity $\mathbf{v}:=\left(v_{1}, v_{2}, v_{3}\right)^{*} \in \mathbf{R}^{3}$ and angular velocity vector $\boldsymbol{\Omega}:=\left(\Omega_{1}, \Omega_{2}, \Omega_{3}\right)^{*} \in \mathbf{R}^{3}$ (both in e) by respectively $\mathbf{v}:=R^{*} \dot{\mathbf{r}}$ and $\hat{\boldsymbol{\Omega}}:=R^{*} \dot{R}$, where for every vector $\mathbf{u}:=\left(u_{1}, u_{2}, u_{3}\right)^{*} \in \mathbf{R}^{3}, \hat{\mathbf{u}}$ is the unique skew-symmetric matrix satisfying

$$
\hat{\mathbf{u}} x:=\mathbf{u} \times x, \quad \forall x \in \mathbf{R}^{3} .
$$

\section{Shape changes}

The shape changes are expressed in the coordinates system given by the body frame $\mathbf{e}$ (i.e. in $x$-coordinates).

In our modeling, the domains occupied by the swimmer are images of the closed unit ball $\bar{B}$ by $C^{1}$ diffeomorphisms, isotopic to the identity, and tending to the identity at infinity, i.e. belonging to to $\mathcal{D}_{0}^{1}\left(\mathbf{R}^{3}\right)$ (the definitions of all of the function spaces are collected in Appendix A).

With these settings, the shape changes over a time interval $[0, T]$ can be simply prescribed by means of functions

$$
t \in[0, T] \mapsto \Theta_{t} \in \mathcal{D}_{0}^{1}\left(\mathbf{R}^{3}\right)
$$




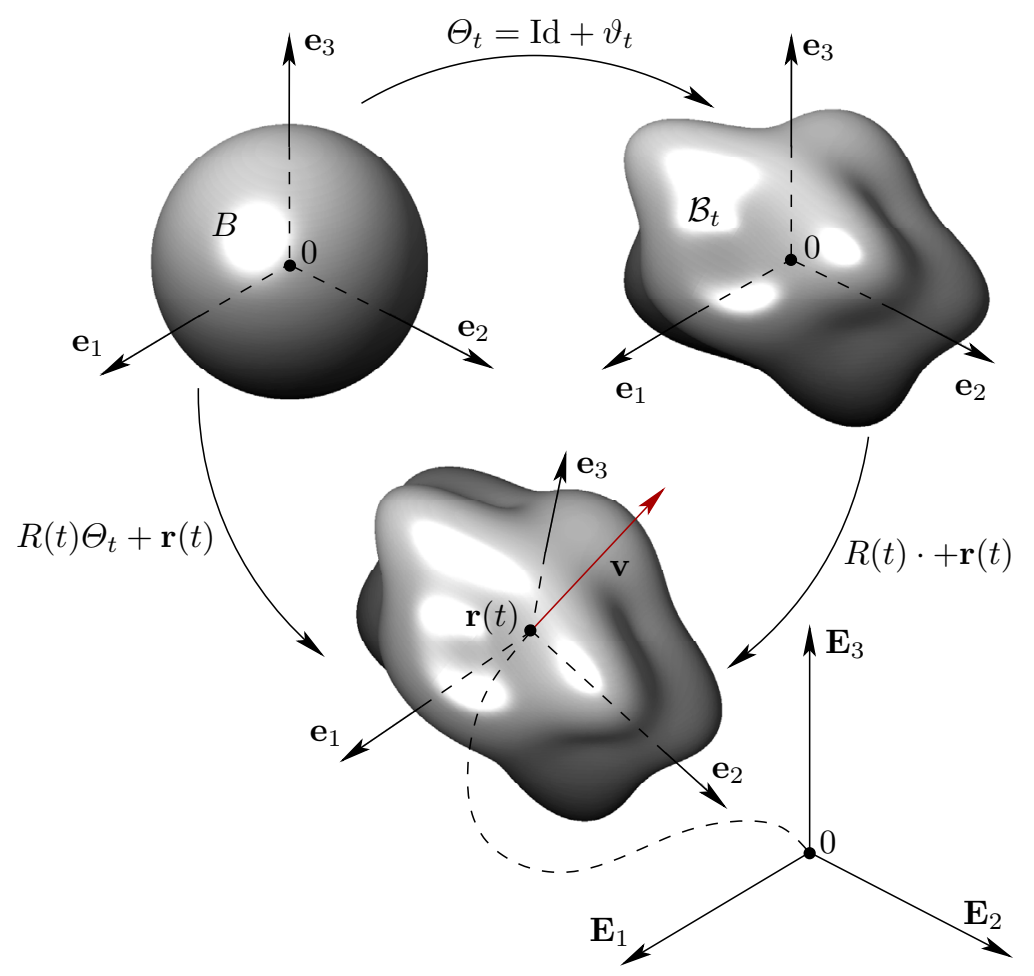

Figure 1. Kinematics of the model: The Galilean frame $\mathbf{E}:=\left(\mathbf{E}_{j}\right)_{1<j \leq 3}$ and the body frame $\mathbf{e}:=\left(\mathbf{e}_{j}\right)_{1 \leq j \leq 3}$ with $\mathbf{e}_{j}=R \mathbf{E}_{j}(R \in \mathrm{SO}(3))$. Quantities are mostly expressed in the body frame. The domain of the body is $\overline{\mathcal{B}}_{t}$ at the time $t$ and $\mathcal{B}_{t}$ is the image of the unit ball $B$ by a diffeomorphism $\Theta_{t}$. The open set $\mathcal{F}_{t}:=\mathbf{R}^{3} \backslash \overline{\mathcal{B}}_{t}$ is the domain of the fluid. The position of the swimmer is given by the vector $\mathbf{r}$ (in $\mathbf{E}$ ) and its orientation by $R \in \mathrm{SO}(3)$. The vector $\mathbf{v}:=R^{*} \dot{\mathbf{r}}$ is the translational velocity (in $\mathbf{e}$ ).

lying in $W^{1,1}\left([0, T], \mathcal{D}_{0}^{1}\left(\mathbf{R}^{3}\right)\right)$. Then, the domain occupied by the swimmer at every time $t \geq 0$ is the closed, bounded, connected set $\overline{\mathcal{B}}_{t}:=\Theta_{t}(\bar{B})$ (keep in mind that we are working in the frame $\mathbf{e}$ ). The swimmer's Eulerian velocity of deformation is

$$
\mathbf{w}_{t}:=\partial_{t} \Theta\left(\Theta^{-1}\right) \text {. }
$$

We shall denote $\Sigma:=\partial B$ the unit ball's boundary while $\Sigma_{t}:=\Theta_{t}(\Sigma)$ will stand for the body-fluid interface. The unit normal vector to $\Sigma_{t}$ directed toward the interior of $\mathcal{B}_{t}$ is $\mathbf{n}_{t}$ and the fluid fills the exterior open set $\mathcal{F}_{t}:=\mathbf{R}^{3} \backslash \overline{\mathcal{B}}_{t}$.

\section{The flow}

The flow is governed by the stationary Stokes equations. Let us denote by $\mathbf{U}$ the Eulerian velocity and by $P$ the pressure in $X$-coordinates, both defined in the fluid domain $R \mathcal{F}_{t}+\mathbf{r}$. They read:

$$
-\mu \Delta \mathbf{U}+\nabla P=0, \quad \nabla \cdot \mathbf{U}=0 \quad \text { in } R \mathcal{F}_{t}+\mathbf{r} \quad(t>0),
$$

where $\mu$ is the viscosity. Introducing for all $x \in \mathcal{F}_{t}$ :

$$
\mathbf{u}(x)=R^{*} \mathbf{U}(R x+\mathbf{r}) \quad \text { and } \quad p(x)=P(R x+\mathbf{r}),
$$


equation (1.2) can be rewritten in the $x$-coordinates as follows:

$$
-\mu \Delta \mathbf{u}+\nabla p=0, \quad \nabla \cdot \mathbf{u}=0 \quad \text { in } \mathcal{F}_{t} \quad(t>0)
$$

Remark 1.1. From now on and unless otherwise indicated, we will work exclusively in the $x$-coordinates.

The equations (1.3) have to be complemented with the no-slip boundary conditions:

$$
\mathbf{u}=\Omega \times x+\mathbf{v}+\mathbf{w}_{t} \text { on } \Sigma_{t},
$$

ensuring the continuity of the velocity field across the swimmer's boundary.

The linearity of these equations leads to introducing the elementary velocities and pressures

$$
\left(\mathbf{u}_{i}, p_{i}\right), \quad(i=1, \ldots, 6) \text { and } \quad\left(\mathbf{u}_{d}, p_{d}\right),
$$

defined as the solutions to the Stokes equations with the boundary conditions

$$
\begin{aligned}
\mathbf{u}_{i} & = \begin{cases}\mathbf{e}_{i} \times x, & i=1,2,3, \\
\mathbf{e}_{i-3}, & i=4,5,6,\end{cases} \\
\text { and } \quad \mathbf{u}_{d} & =\mathbf{w}_{t} \text { on } \Sigma_{t} .
\end{aligned}
$$

Then, the velocity $\mathbf{u}$ and the pressure $p$ can be decomposed as

$$
\begin{aligned}
\mathbf{u} & =\sum_{i=1}^{3} \Omega_{i} \mathbf{u}_{i}+\sum_{i=4}^{6} v_{i-3} \mathbf{u}_{i}+\mathbf{u}_{d} \\
\text { and } \quad p & =\sum_{i=1}^{3} \Omega_{i} p_{i}+\sum_{i=4}^{6} v_{i-3} p_{i}+p_{d} \text { in } \mathcal{F}_{t} .
\end{aligned}
$$

Notice that the pairs $\left(\mathbf{u}_{i}, p_{i}\right)(i=1, \ldots, 6)$ and $\left(\mathbf{u}_{d}, p_{d}\right)$ are well-defined in the weighted Sobolev spaces $\left(W_{0}^{1}\left(\mathcal{F}_{t}\right)\right)^{3} \times L^{2}\left(\mathcal{F}_{t}\right)($ see the Appendix A).

\section{Dynamics}

As already pointed out before, for microswimmers, the inertia effects are neglected in the modeling. Newton's laws reduce to

$$
\begin{aligned}
\int_{\Sigma_{t}} x \times \mathbb{T}(\mathbf{u}, p) \mathbf{n}_{t} \mathrm{~d} \sigma & =\mathbf{0} \quad \text { (balance of torque) } \\
\int_{\Sigma_{t}} \mathbb{T}(\mathbf{u}, p) \mathbf{n}_{t} \mathrm{~d} \sigma & =\mathbf{0} \quad \text { (balance of force) }
\end{aligned}
$$

where

$$
\mathbb{T}(\mathbf{u}, p):=2 \mu D(\mathbf{u})-p \mathrm{Id}
$$

is the stress tensor of the fluid, with $D(\mathbf{u}):=\left(\nabla \mathbf{u}+\nabla \mathbf{u}^{*}\right) / 2$.

The stress tensor is linear with respect to $(\mathbf{u}, p)$ so it can be decomposed into

$$
\mathbb{T}(\mathbf{u}, p)=\sum_{i=1}^{3} \Omega_{i} \mathbb{T}\left(\mathbf{u}_{i}, p_{i}\right)+\sum_{i=4}^{6} v_{i-3} \mathbb{T}\left(\mathbf{u}_{i}, p_{i}\right)+\mathbb{T}\left(\mathbf{u}_{d}, p_{d}\right) .
$$


In order to rewrite Newton's laws in a compact form, we introduce the $6 \times 6$ matrix $\mathbb{M}(t)$ whose entries are

$$
M_{i j}(t):= \begin{cases}\int_{\Sigma_{t}}\left(x \times \mathbf{e}_{i}\right) \cdot \mathbb{T}\left(\mathbf{u}_{j}, p_{j}\right) \mathbf{n}_{t} \mathrm{~d} \sigma & (1 \leq i \leq 3,1 \leq j \leq 6) ; \\ \int_{\Sigma_{t}} \mathbf{e}_{i-3} \cdot \mathbb{T}\left(\mathbf{u}_{j}, p_{j}\right) \mathbf{n}_{t} \mathrm{~d} \sigma & (4 \leq i \leq 6,1 \leq j \leq 6)\end{cases}
$$

and $\mathbf{N}(t)$, the vector of $\mathbf{R}^{6}$ whose entries are

$$
N_{i}(t):= \begin{cases}\int_{\Sigma_{t}}\left(x \times \mathbf{e}_{i}\right) \cdot \mathbb{T}\left(\mathbf{u}_{d}, p_{d}\right) \mathbf{n}_{t} \mathrm{~d} \sigma & (1 \leq i \leq 3) \\ \int_{\Sigma_{t}} \mathbf{e}_{i-3} \cdot \mathbb{T}\left(\mathbf{u}_{d}, p_{d}\right) \mathbf{n}_{t} \mathrm{~d} \sigma & (4 \leq i \leq 6) .\end{cases}
$$

For the statement of the optimal control result, we will also need the quantity:

$$
A(t):=\int_{\Sigma_{t}} \mathbf{w}_{t} \cdot \mathbb{T}\left(\mathbf{u}_{d}, p_{d}\right) \mathbf{n}_{t} \mathrm{~d} \sigma
$$

With these settings, Newton's laws (1.5) take the convenient form

$$
\mathbb{M}(t)(\Omega, \mathbf{v})^{*}+\mathbf{N}(t)=0 .
$$

Upon an integration by parts, the entries of the matrix $\mathbb{M}(t)$ can be rewritten as

$$
M_{i j}(\mathbf{s}):=2 \mu \int_{\mathcal{F}_{t}} D\left(\mathbf{u}_{i}\right): D\left(\mathbf{u}_{j}\right) \mathrm{d} x
$$

whence we deduce that $\mathbb{M}(t)$ is symmetric and positive definite. We infer that the swimming motion is governed by the equation:

$$
\left(\begin{array}{c}
\boldsymbol{\Omega} \\
\mathbf{v}
\end{array}\right)=-\mathbb{M}(t)^{-1} \mathbf{N}(t), \quad(0 \leq t \leq T)
$$

To determine the rigid motion in the fixed frame $\mathbf{E}$, equation (1.7a) has to be supplemented with the ODE:

$$
\frac{\mathrm{d}}{\mathrm{d} t}\left(\begin{array}{c}
R \\
\mathbf{r}
\end{array}\right)=\left(\begin{array}{c}
R \hat{\Omega} \\
R \mathbf{v}
\end{array}\right), \quad(0<t<T),
$$

together with Cauchy data for $R(0)$ and $\mathbf{r}(0)$. At this point, we can identify the control as being the function $t \in[0, T] \mapsto \Theta_{t} \in \mathcal{D}_{0}^{1}\left(\mathbf{R}^{3}\right)$.

Notice that the dependence of the dynamics in the control is strongly nonlinear. Indeed $\Theta_{t}$ describes the shape of the body and hence also the domain of the fluid in which are set the PDEs of the elementary velocity fields involved in the expressions of the matrices $\mathbb{M}(t)$ and $\mathbf{N}(t)$.

Considering (1.7), we deduce:

Proposition 1.2. The dynamics (1.7) of a microswimmer is independent of the viscosity of the fluid. Or, in other words, the same shape changes produce the same rigid displacement, whatever the viscosity of the fluid is.

Proof. Let $\left(\mathbf{u}_{j}, p_{j}\right)$ be an elementary solution (as defined in the modeling above) to the Stokes equations corresponding to the viscosity $\mu>0$, then $\left(\mathbf{u}_{j},(\tilde{\mu} / \mu) p_{j}\right)$ is the same elementary solution corresponding to the viscosity $\tilde{\mu}>0$. Since the Euler-Lagrange equation (1.7) depends only on the Eulerian velocities $\mathbf{u}_{j}$, the proof is completed.

As a consequence of this Proposition we will set $\mu=1$ in the sequel. 


\section{Gauge fixing}

As we will see later on in Proposition 1.6, system (1.7) is well-posed for any shape function

$$
\Theta \in W^{1,1}\left([0, T], \mathcal{D}_{0}^{1}\left(\mathbf{R}^{3}\right)\right) .
$$

It means that any $\Theta \in W^{1,1}\left([0, T], \mathcal{D}_{0}^{1}\left(\mathbf{R}^{3}\right)\right)$ used as control in the dynamics (1.7) will produce a unique absolutely continuous rigid motion $t \in[0, T] \mapsto(R(t), \mathbf{r}(t)) \in \mathrm{SO}(3) \times \mathbf{R}^{3}$.

Proposition 1.3. Let $\Theta, \Theta^{\dagger} \in W^{1,1}\left([0, T], \mathcal{D}_{0}^{1}\left(\mathbf{R}^{3}\right)\right)$ be two control functions such that $\left.\Theta\right|_{t=0}=\left.\Theta\right|_{t=0} ^{\dagger}$ and which differ up to a rigid displacement on the unit sphere for every $t>0$, i.e. for every $t \in[0, T]$, there exists $(Q(t), \mathbf{s}(t)) \in \mathrm{SO}(3) \times \mathbf{R}^{3}$ such that

$$
(Q(0), \mathbf{s}(0))=(\operatorname{Id}, \mathbf{0}) \quad \text { and }\left.\quad \Theta_{t}^{\dagger}\right|_{\Sigma}=\left.Q(t) \Theta_{t}\right|_{\Sigma}+\mathbf{s}(t) .
$$

We denote respectively by

$$
\begin{aligned}
& t \in[0, T] \mapsto(R(t), \mathbf{r}(t)) \in \mathrm{SO}(3) \times \mathbf{R}^{3}, \\
& t \in[0, T] \mapsto\left(R^{\dagger}(t), \mathbf{r}^{\dagger}(t)\right) \in \mathrm{SO}(3) \times \mathbf{R}^{3},
\end{aligned}
$$

the solutions to system (1.7) with the same Cauchy data $\left(R_{0}, \mathbf{r}_{0}\right) \in \mathrm{SO}(3) \times \mathbf{R}^{3}$ and with respectively the controls $\Theta$ and $\Theta^{\dagger}$. Then we get the identity:

$$
\left(R^{\dagger}(t), \mathbf{r}^{\dagger}(t)\right):=\left(R(t) Q(t)^{*}, \mathbf{r}(t)-R(t) Q(t)^{*} \mathbf{s}(t)\right), \quad \forall t \in[0, T] .
$$

In particular

$$
R^{\dagger}(t) \Theta_{t}^{\dagger}+\mathbf{r}^{\dagger}(t)=R(t) \Theta_{t}+\mathbf{r}(t) \quad \forall t \in[0, T]
$$

(i.e. the swimmer's global motion is the same in both cases).

This proposition tells us that two shape changes whose restrictions on the unit sphere differ only up to a rigid displacement will produce the exact same global motion of the swimmer.

It is worth remarking that if we apply the proposition with $\Theta$ constant in time (the boundary of the swimmer is therefore $\Theta(\Sigma)$ at every time), we can deduce that any shape change which reduces to a rigid deformation $Q(t) x+\mathbf{s}(t)$ on the swimmer's boundary $\Theta(\Sigma)$ will produce a displacement $\left(Q(t)^{*},-Q(t)^{*} \mathbf{s}(t)\right)$. The resulting global motion obtained by composing the shape changes with the rigid motion is then

$$
Q^{*}(t)(Q(t) \Theta(x)+\mathbf{s}(t))-Q(t)^{*} \mathbf{s}(t)=\Theta(x) \quad \forall x \in \Sigma, \quad \forall t \in[0, T]
$$

which means that the swimmer is actually motionless (the rigid deformation of the swimmer's boundary used as control is exactly counterbalanced by its rigid displacement provided by the dynamics). In this case, at least the exact controllability problem turns out to be trivial. Indeed, for the dynamics to produce the rigid motion $(Q(t), \mathbf{s}(t))$, it suffices to use a deformation whose restriction on $\Theta(\Sigma)$ is the rigid motion $\left(Q(t)^{*},-Q(t)^{*} \mathbf{s}(t)\right)$.

These observations stress the fact that gauge conditions have to be added in order to single out a representative among every class of equivalent controls (i.e. that differ up to a rigid displacement on the unit sphere and hence produce the same global swimmer's motion).

What happens inside the swimmer is irrelevant regarding its locomotion, so the gauge condition has to bear upon the restriction of $\Theta$ on the unit sphere only.

Inspired by the so-called self-propelled constraints for weighted swimmers (as explained in [5]), we propose that a shape function, to be an allowable control, satisfies the following identities:

$$
\begin{aligned}
\int_{\Sigma} \Theta_{t}(x) \mathrm{d} \sigma=\mathbf{0} & \text { (for all } t \in[0, T]) \\
\int_{\Sigma} \partial_{t} \Theta_{t}(x) \times \Theta_{t}(x) \mathrm{d} \sigma=\mathbf{0} & \text { (for a.e. } t \in[0, T]) .
\end{aligned}
$$


Notice in particular that no shape function whose restriction on the unit sphere $\Sigma$ is a rigid deformation (other than the identity) satisfies these conditions.

About the existence of such deformations, we have in particular:

Proposition 1.4. For every function $\Theta$ in $W^{1,1}\left([0, T], \mathcal{D}_{0}^{1}\left(\mathbf{R}^{3}\right)\right)$ such that

$$
\int_{\Sigma} \Theta_{t=0}(x) \mathrm{d} \sigma=\mathbf{0}
$$

there exists a function $\Theta^{\dagger}$ in $W^{1,1}\left([0, T], \mathcal{D}_{0}^{1}\left(\mathbf{R}^{3}\right)\right)$ satisfying (1.8) and an unique absolutely continuous rigid displacement

$$
t \in[0, T] \mapsto(Q(t), \mathbf{s}(t)) \in \mathrm{SO}(3) \times \mathbf{R}^{3}
$$

such that $Q(0)=\mathrm{Id}, \mathbf{s}(0)=\mathbf{0}$ and $\left.\Theta_{t}^{\dagger}\right|_{\Sigma}=\left.\left(Q(t) \Theta_{t}+\mathbf{s}(t)\right)\right|_{\Sigma}$ for every $t \in[0, T]$.

In other words, any function of $W^{1,1}\left([0, T], \mathcal{D}_{0}^{1}\left(\mathbf{R}^{3}\right)\right)$ satisfying the first equality of (1.8) at $t=0$ is allowable (in the sense that it satisfies (1.8)) up to a composition by a suitable rigid deformation.

Definition 1.5. We denote by $\mathcal{A}$ the non-empty closed subset of $W^{1,1}\left([0, T], \mathcal{D}_{0}^{1}\left(\mathbf{R}^{3}\right)\right)$ consisting of all of the functions verifying (1.8).

\subsection{Main results}

The first result ensures the well posedness of system (1.7) and the second is about the continuity of the input-output mapping:

Proposition 1.6. For any $T>0$ and

- every function $\Theta \in W^{1,1}\left([0, T], \mathcal{D}_{0}^{1}\left(\mathbf{R}^{3}\right)\right)$ (respectively of class $\left.C^{p}, p=1, \ldots,+\infty, \omega\right)$;

- every initial data $(R(0), \mathbf{r}(0)) \in \mathrm{SO}(3) \times \mathbf{R}^{3}$;

System (1.7) admits a unique solution $t \in[0, T] \mapsto(R(t), \mathbf{r}(t)) \in \mathrm{SO}(3) \times \mathbf{R}^{3}$ (in the sense of Carathéodory) absolutely continuous on $[0, T]$ (respectively of class $\left.C^{p}\right)$.

Notice that this proposition improves the results of [13]. Indeed in [13], the shape function has to be (with our notation) in $\operatorname{Lip}\left([0, T], \mathcal{D}_{0}^{1}\left(\mathbf{R}^{3}\right)\right) \cap L^{\infty}\left([0, T], C^{2}\left(\mathbf{R}^{3}\right)^{3}\right)$.

Proposition 1.7. - Let $\left(\Theta_{j}\right)_{j \geq 1} \subset W^{1,1}\left([0, T], \mathcal{D}_{0}^{1}\left(\mathbf{R}^{3}\right)\right)$ be a sequence of controls converging to a function $\bar{\Theta}$.

- Let a pair $\left(R_{0}, \mathbf{r}_{0}\right) \in \mathrm{SO}(3) \times \mathbf{R}^{3}$ be given;

and denote by

$$
t \in[0, T] \mapsto(\bar{R}(t), \overline{\mathbf{r}}(t)) \in \mathrm{SO}(3) \times \mathbf{R}^{3}
$$

the solution in $A C\left([0, T], \mathrm{SO}(3) \times \mathbf{R}^{3}\right)$ to system (1.7) with control $\bar{\Theta}$ and Cauchy data $\left(R_{0}, \mathbf{r}_{0}\right)$. Then, the unique solution $\left(R^{j}, \mathbf{r}^{j}\right)$ to system (1.7) with control $\Theta^{j}$ and Cauchy data $\left(R_{0}, \mathbf{r}_{0}\right)$ converges in $A C\left([0, T], \operatorname{SO}(3) \times \mathbf{R}^{3}\right)$ to $(\bar{R}, \overline{\mathbf{r}})$ as $j \rightarrow+\infty$.

We denote by $\mathrm{M}(3)$ the Banach space of the $3 \times 3$ matrices endowed with any matrix norm. The main result of this article addresses the controllability of system (1.7):

Theorem 1.8 (Synchronized Swimming). Assume that the following data are given:

1. A function $\bar{\Theta} \in \mathcal{A}$ (the reference shape changes);

2. A continuous function $t \in[0, T] \mapsto(\bar{R}(t), \overline{\mathbf{r}}(t)) \in \mathrm{SO}(3) \times \mathbf{R}^{3}$ (the reference trajectory to be followed).

Then, for any $\varepsilon>0$, there exists a function $t \in[0, T] \mapsto \Theta_{t} \in \mathcal{D}_{0}^{1}\left(\mathbf{R}^{3}\right)$ (the actual shape changes) in $\mathcal{A}$, which can be chosen analytic, such that 
1. $\Theta_{0}=\bar{\Theta}_{0}, \Theta_{T}=\bar{\Theta}_{T}$;

2. $\sup _{t \in[0, T]}\left(\left\|\bar{\Theta}_{t}-\Theta_{t}\right\|_{C_{0}^{1}\left(\mathbf{R}^{3}\right)^{3}}+\|\bar{R}(t)-R(t)\|_{\mathrm{M}(3)}+\|\overline{\mathbf{r}}(t)-\mathbf{r}(t)\|_{\mathbf{R}^{3}}\right)<\varepsilon ;$

where the function $t \in[0, T] \mapsto(R(t), \mathbf{r}(t)) \in \mathrm{SO}(3) \times \mathbf{R}^{3}$ is the unique solution to system (1.7) with initial data $(R(0), \mathbf{r}(0))=(\bar{R}(0), \overline{\mathbf{r}}(0))$ and control $\Theta$.

This theorem tells us that any 3D microswimmer undergoing approximately any prescribed shape changes can approximately track by swimming any given trajectory. It may seem surprising that the shape changes, which are supposed to be the control of our problem, can also be somehow preassigned. Actually, the trick is that they can only be approximately prescribed. We are going to show that arbitrarily small superimposed shape changes suffice for controlling the swimming motion.

When no macro shape changes are preassigned we have:

Theorem 1.9 (Freestyle swimming). Assume that the following data are given:

1. A function $\bar{\Theta}_{\mathrm{rst}} \in \mathcal{D}_{0}^{1}\left(\mathbf{R}^{3}\right)$ such that $\int_{\Sigma} \bar{\Theta}_{\mathrm{rst}} \mathrm{d} \sigma=0$ (the reference shape at rest);

2. A continuous function $t \in[0, T] \mapsto(\bar{R}(t), \overline{\mathbf{r}}(t)) \in \mathrm{SO}(3) \times \mathbf{R}^{3}$ (the reference trajectory);

Then, for any $\varepsilon>0$ there exists a function $\Theta_{\mathrm{rst}} \in \mathcal{D}_{0}^{1}\left(\mathbf{R}^{3}\right)$ (the actual shape at rest) enjoying the following properties:

1. $\int_{\Sigma} \Theta_{\mathrm{rst}} \mathrm{d} \sigma=\mathbf{0}$;

2. $\left\|\bar{\Theta}_{\mathrm{rst}}-\Theta_{\mathrm{rst}}\right\|_{C_{0}^{1}\left(\mathbf{R}^{3}\right)^{3}}<\varepsilon$;

3. for almost any 4-uplet $\left(\mathbf{V}_{1}, \ldots, \mathbf{V}_{4}\right) \in\left(C_{0}^{1}\left(\mathbf{R}^{3}\right)^{3}\right)^{4}$ satisfying

(a) $\int_{\Sigma} \mathbf{V}_{i} \mathrm{~d} x=\mathbf{0}$;

(b) $\int_{\Sigma} \Theta_{\mathrm{rst}} \times \mathbf{V}_{i} \mathrm{~d} \sigma=\mathbf{0}$;

(c) $\int_{\Sigma} \mathbf{V}_{i} \times \mathbf{V}_{j} \mathrm{~d} \sigma=\mathbf{0}(i, j=1, \ldots, 4)$;

there exists a function

$$
t \in[0, T] \mapsto s(t):=\left(s_{1}(t), \ldots, s_{4}(t)\right)^{*} \in \mathbf{R}^{4}
$$

(which can be chosen analytic) such that, using

$$
\Theta_{t}:=\Theta_{\mathrm{rst}}+\sum_{i=1}^{4} s_{i}(t) \mathbf{V}_{i} \in \mathcal{D}_{0}^{1}\left(\mathbf{R}^{3}\right)
$$

as control in the dynamics (1.7), we get

$$
\sup _{t \in[0, T]}\left(\|\bar{R}(t)-R(t)\|_{\mathrm{M}(3)}+\|\overline{\mathbf{r}}(t)-\mathbf{r}(t)\|_{\mathbf{R}^{3}}\right)<\varepsilon
$$

where the function $t \in[0, T] \mapsto(R(t), \mathbf{r}(t)) \in \mathrm{SO}(3) \times \mathbf{R}^{3}$ is the unique solution to ODEs (1.7) with initial data $(R(0), \mathbf{r}(0))=(\bar{R}(0), \overline{\mathbf{r}}(0))$.

We claim in this Theorem that any 3D microswimmer (maybe up to an arbitrarily small modification of its initial shape) is able to swim by means of allowable deformations (i.e. satisfying the constraints (1.8)) obtained as a suitable combination of almost any given four basic movements.

In order to state now the result about the existence of optimal control functions, we have to particularize somehow the form of the control. So, for

- every $T>0$;

- every compact, convex set $K \subset \mathbf{R}^{n}$;

- every $\Theta_{\mathrm{rst}} \in \mathcal{D}_{0}^{1}\left(\mathbf{R}^{3}\right)$ such that $\int_{\Sigma} \Theta_{\mathrm{rst}} \mathrm{d} x=\mathbf{0}$;

- every family $\mathcal{V}:=\left(\mathbf{V}_{1}, \ldots, \mathbf{V}_{n}\right) \in\left(C_{0}^{1}\left(\mathbf{R}^{3}\right)^{3}\right)^{n}$ of $n$ vector fields satisfying 
1. $\int_{\Sigma} \mathbf{V}_{i} \mathrm{~d} x=\mathbf{0}$;

2. $\int_{\Sigma} \Theta_{\mathrm{rst}} \times \mathbf{V}_{i} \mathrm{~d} x=\mathbf{0}$;

3. $\int_{\Sigma} \mathbf{V}_{i} \times \mathbf{V}_{j} \mathrm{~d} x=\mathbf{0}(i, j=1, \ldots, n)$.

We denote by $\mathcal{U}\left(T, K, \Theta_{\text {rst }}, \mathcal{V}\right)$ the set of all the control functions $\Theta_{t} \in W^{1,1}\left([0, T], \mathcal{D}_{0}^{1}\left(\mathbf{R}^{3}\right)\right)$ having the form

$$
\Theta_{t}=\Theta_{\mathrm{rst}}+\sum_{i=1}^{n} s_{i}(t) \mathbf{V}_{i}
$$

where

1. $t \in[0, T] \mapsto s(t):=\left(s_{1}(t), \ldots, s_{n}(t)\right)^{*} \in \mathbf{R}^{n}$ is in $A C\left([0, T], \mathbf{R}^{n}\right)$;

2. $\dot{s}(t) \in K$ for a.e. $t \in[0, T]$.

For every $\left(R_{0}, \mathbf{r}_{0}, \Theta_{0}\right) \in \mathrm{SO}(3) \times \mathbf{R}^{3} \times \mathcal{D}_{0}^{1}\left(\mathbf{R}^{3}\right)$ and every $T>0$, we define also $\chi\left(T, K, \Theta_{\text {rst }}, \mathcal{V}, R_{0}, \mathbf{r}_{0}, \Theta_{0}\right)$ as the set consisting of all the triplets $(\bar{R}, \overline{\mathbf{r}}, \bar{\Theta}) \in \mathrm{SO}(3) \times \mathbf{R}^{3} \times \mathcal{D}_{0}^{1}\left(\mathbf{R}^{3}\right)$ for which there exists a control function $\Theta_{t} \in \mathcal{U}\left(T, K, \Theta_{\mathrm{rst}}, \mathcal{V}\right)$, satisfying $\Theta_{t=0}=\Theta_{0}, \Theta_{t=T}=\bar{\Theta}$ and steering the dynamics (1.7) from $\left(R_{0}, \mathbf{r}_{0}\right)($ at $t=0)$ to $(\bar{R}, \overline{\mathbf{r}})($ at $t=T)$.

The following result holds:

Theorem 1.10 (Existence of an optimal control for short time interval).

- For every continuous function $f: \mathrm{SO}(3) \times \mathbf{R}^{3} \times \mathcal{D}_{0}^{1}\left(\mathbf{R}^{3}\right) \times C_{0}^{1}\left(\mathbf{R}^{3}\right)^{3} \rightarrow \mathbf{R}$, convex in the fourth variable;

- For every data $K, \Theta_{\mathrm{rst}}, \mathcal{V}, R_{0}, \mathbf{r}_{0}, \Theta_{0}$ as described above;

there exists a time $T^{*}>0$ such that, for all $0<T \leq T^{*}$ and for all $\left(R_{1}, \mathbf{r}_{1}, \Theta_{1}\right) \in \chi\left(T, K, \Theta_{\mathrm{rst}}, \mathcal{V}, R_{0}, \mathbf{r}_{0}, \Theta_{0}\right)$, there exists a control function realizing the minimum of the cost

$$
\int_{0}^{T} f\left(R(t), \mathbf{r}(t), \Theta_{t}, \partial_{t} \Theta_{t}\right) \mathrm{d} t
$$

among the controls $\Theta$ in $\mathcal{U}\left(T, K, \Theta_{\mathrm{rst}}, \mathcal{V}\right)$ satisfying $\Theta_{t=0}=\Theta_{0}, \Theta_{t=T}=\Theta_{1}$ and steering the dynamics (1.7) from $\left(R_{0}, \mathbf{r}_{0}\right)$ (at $\left.t=0\right)$ to $\left(R_{1}, \mathbf{r}_{1}\right)($ at $t=T)$.

The following observations are worth taking into account:

- The theorem applies only for control functions living in a finite dimensional space.

- The hypothesis ensuring that $\dot{s}$ is valued in a compact set is quite natural. It means that the rate of shape changes has to remain bounded.

- The short time hypothesis is natural too. It is necessary to prevent the swimmer from self-deforming "to much". For instance, one can imagine that to indefinitely improve its efficiency in swimming in one direction, a swimmer could make its shape become more and more singular (for instance very elongated and thin or very flat). These phenomena would prevent optimal deformations from existing.

- If we set $\Theta_{1}=\Theta_{0}$, then the theorem ensures the existence of optimal strokes (i.e. periodic deformations).

An example of cost functional satisfying the assumptions of this Theorem is:

$$
J:=\int_{0}^{T}\left(\int_{\mathcal{F}_{t}} D(\mathbf{u}): D(\mathbf{u}) \mathrm{d} x\right) \mathrm{d} t,
$$

in which $(\mathbf{u}, p)$ is defined in (1.4). It measures the viscous energy dissipated in the fluid. Upon an integration by parts, $J$ can be rewritten as:

$$
J=\frac{1}{2} \int_{0}^{T} \mathbf{u}(t) \cdot\left(\int_{\Sigma_{t}} \mathbb{T}(\mathbf{u}, p) \mathbf{n}_{t} \mathrm{~d} \sigma\right) \mathrm{d} t
$$


Notice that $J$ can also be interpretable as the work of the hydrodynamical forces exerted on the swimmer over the time interval $[0, T]$ and is similar to the cost function used in [2]. Using the matrices and vectors defined in (1.6), and the dynamics (1.7a), we get:

$$
J=\frac{1}{2} \int_{0}^{T}\left(A(t)-\mathbf{N}(t)^{t} \mathbb{M}(t)^{-1} \mathbf{N}(t)\right) \mathrm{d} t .
$$

In this form and according to the regularity results that will be proved later on for the functions $t \in[0, T] \mapsto A(t)$, $t \in[0, T] \mapsto \mathbb{M}(t)$ and $t \in[0, T] \mapsto \mathbf{N}(t)$, it can be verified that Theorem 1.10 applies.

The proofs of the results stated in this section rely on the following leading ideas:

First, we shall identify a set of parameters necessary to thoroughly characterize a swimmer and its way of swimming (these parameters are its shape and a finite number of basic movements, satisfying the constraints (1.8)). Any set of such parameters will be termed a swimmer signature (denoted SS in short). Then, the set of all of the SS will be shown to be an (infinite dimensional) analytic connected embedded submanifold of a Banach space.

The second step of the reasoning will consist in proving that the swimmer's ability to track any given trajectory (while undergoing approximately any preassigned shape changes) is related to the vanishing of some analytic functions depending on the SS. These functions are connected to the determinant of some vector fields and their Lie brackets (we will invoke classical results of Geometric Control Theory). By direct calculation, we will prove that at least one swimmer (corresponding to one particular SS) has this ability. An elementary property of analytic functions will allow us to conclude that almost any SS (or equivalently any microswimmer) has this property.

Eventually, the existence of an optimal control in Theorem 1.10 is a straightforward consequence of Filippov Theorem (see [1], Chap. 10)

\subsection{Outline of the paper}

The next Section is dedicated to the notion of swimmer signature (definition and properties). In Section 3 we show that the matrix $\mathbb{M}(t)$ and the vector $\mathbf{N}(t)$ (in (1.7a)) are analytic functions in the SS (swimmer signature, seen as a variable) and in Section 4 we will restate the control problem in order to fit with the general framework of Geometric Control Theory. In the same Section, a particular case of swimmer will be shown to be controllable. In Section 5 the proof of the main results will be carried out. Section 6 contains some words of conclusion. Technical results and definitions are gathered in the appendix in order to make the paper more readable.

\section{SWIMMER SIGNATURE}

A swimmer signature is a set of parameters characterizing swimmers whose deformations consist in a combination of a finite number of basic movements.

Definition 2.1. For any positive integer $n$, we denote by $\mathcal{C}(n)$ the subset of

$$
\mathcal{D}_{0}^{1}\left(\mathbf{R}^{3}\right) \times\left(C_{0}^{1}\left(\mathbf{R}^{3}\right)^{3}\right)^{n}
$$

consisting of all of the pairs $c:=\left(\Theta_{\text {rst }}, \mathcal{V}\right)$ such that, denoting $\mathcal{V}:=\left(\mathbf{V}_{1}, \ldots, \mathbf{V}_{n}\right)$, the following conditions hold

1. the set $\left\{\left.\mathbf{V}_{i}\right|_{\Sigma} \cdot \mathbf{e}_{k}, 1 \leq i \leq n, k=1,2,3\right\}$ is a free family in $C^{1}(\Sigma)$;

2. every pair $\left(\mathbf{V}, \mathbf{V}^{\prime}\right)$ of elements of $\left\{\Theta_{\text {rst }}, \mathbf{V}_{1}, \ldots, \mathbf{V}_{n}\right\}$ satisfies $\int_{\Sigma} \mathbf{V} \mathrm{d} x=\mathbf{0}$ and $\int_{\Sigma} \mathbf{V} \times \mathbf{V}^{\prime} \mathrm{d} x=\mathbf{0}$.

We call swimmer signature (SS in short) any element $c$ of $\mathcal{C}(n)$. 
By definition, $\mathcal{D}_{0}^{1}\left(\mathbf{R}^{3}\right)$ is open in the affine space $\{\operatorname{Id}\}+C_{0}^{1}\left(\mathbf{R}^{3}\right)^{3}$ (see Appendix A). We deduce that for any $c \in \mathcal{C}(n)$, the set

$$
\left\{s:=\left(s_{1}, \ldots, s_{n}\right)^{*} \in \mathbf{R}^{n}: \Theta_{\mathrm{rst}}+\sum_{i=1}^{n} s_{i} \mathbf{V}_{i} \in \mathcal{D}_{0}^{1}\left(\mathbf{R}^{3}\right)\right\}
$$

is open as well in $\mathbf{R}^{n}$ and we denote $\mathcal{S}(c)$ its connected component containing $s=0$.

Definition 2.2. For any positive integer $n$, we call swimmer full signature (SFS in short) any pair $\mathbf{c}:=(c, s)$ such that $c \in \mathcal{C}(n)$ and $s \in \mathcal{S}(c)$. We denote $\mathcal{C}_{F}(n)$ the set of all of these pairs.

Restatement of the problem in terms of swimmer signature (SS) and swimmer full signature (SFS)

Pick a SS, $c=\left(\Theta_{\text {rst }}, \mathcal{V}\right) \in \mathcal{C}(n)$ with $\mathcal{V}:=\left(\mathbf{V}_{1}, \ldots, \mathbf{V}_{n}\right)$ (for some integer $n$ ). Denote, for all $s \in \mathcal{S}(c)$,

$$
\Theta_{s}:=\Theta_{\mathrm{rst}}+\sum_{i=1}^{n} s_{i} \mathbf{V}_{i}
$$

$\left(\mathbf{c}:=(c, s) \in \mathcal{C}(n)\right.$ is hence a SFS). The body of the swimmer occupies the domain $\overline{\mathcal{B}}:=\Theta_{\text {rst }}(\bar{B})$ at rest and $\overline{\mathcal{B}}_{\mathbf{c}}:=\Theta_{s}(\bar{B})$ (for any $s \in \mathcal{S}(c)$ ) when swimming.

Notice that within this construction, the shape changes on a time interval $[0, T](T>0)$ are merely given through an absolutely continuous function

$$
t:[0, T] \mapsto s(t) \in \mathcal{S}(c) .
$$

If $t \in[0, T] \mapsto \dot{s}(t) \in \mathbf{R}^{n}$ stands for its time derivative in $L^{1}\left([0, T], \mathbf{R}^{n}\right)$, the Lagrangian velocity at a point $x$ of $\bar{B}$ is

$$
\sum_{i=1}^{n} \dot{s}_{i}(t) \mathbf{V}_{i}(x)
$$

while the Eulerian velocity at a point $x \in \overline{\mathcal{B}}_{\mathbf{c}}$ is

$$
\sum_{i=1}^{n} \dot{s}_{i}(t) \mathbf{w}_{s}^{i}(x) \quad \text { with } \quad \mathbf{w}_{s}^{i}(x):=\mathbf{V}_{i}\left(\Theta_{s}^{-1}(x)\right) .
$$

Due to assumption 2 of Definition 2.1, the constraints (1.8) are automatically satisfied.

The elementary fluid velocities and elementary pressure functions corresponding to the rigid motions depend only on the SFS. Therefore, they will be denoted in the sequel $\mathbf{u}_{i}(\mathbf{c})$ and $p_{i}(\mathbf{c})$ to emphasize this dependence. The same remark holds for the matrix $\mathbb{M}(t)$ whose notation is turned into $\mathbb{M}(\mathbf{c})$. The elementary velocity and pressure $\left(\mathbf{u}_{d}, p_{d}\right)$ connected to the shape changes can be decomposed into

$$
\begin{aligned}
\mathbf{u}_{d} & =\sum_{i=1}^{n} \dot{s}_{i} \mathbf{w}_{i}(\mathbf{c}) \\
p_{d} & =\sum_{i=1}^{n} \dot{s}_{i} \pi_{i}(\mathbf{c}) .
\end{aligned}
$$

In this sum, each pair $\left(\mathbf{w}_{i}(\mathbf{c}), \pi_{i}(\mathbf{c})\right)$ solves the Stokes equations in $\mathcal{F}_{\mathbf{c}}:=\mathbf{R}^{3} \backslash \overline{\mathcal{B}}_{\mathbf{c}}$ with boundary conditions $\mathbf{w}_{i}(\mathbf{c})=\mathbf{w}_{s}^{i}$ on $\Sigma_{\mathbf{c}}:=\partial \mathcal{B}_{\mathbf{c}}$.

Introducing the matrix $\mathbb{N}(\mathbf{c})$, whose elements are

$$
N_{i j}(\mathbf{c}):= \begin{cases}\int_{\Sigma_{\mathbf{c}}}\left(x \times \mathbf{e}_{i}\right) \cdot \mathbb{T}\left(\mathbf{w}_{j}(\mathbf{c}), \pi_{j}(\mathbf{c})\right) \mathbf{n} \mathrm{d} \sigma & (1 \leq i \leq 3,1 \leq j \leq n) ; \\ \int_{\Sigma_{\mathbf{c}}} \mathbf{e}_{i-3} \cdot \mathbb{T}\left(\mathbf{w}_{j}(\mathbf{c}), \pi_{j}(\mathbf{c})\right) \mathbf{n} \mathrm{d} \sigma & (4 \leq i \leq 6,1 \leq j \leq n) ;\end{cases}
$$


(recall that the viscosity $\mu$ can be chosen equal to 1), the dynamics (1.7a) can now be rewritten in the form:

$$
\left(\begin{array}{c}
\Omega \\
\mathbf{v}
\end{array}\right)=-\mathbb{M}(\mathbf{c})^{-1} \mathbb{N}(\mathbf{c}) \dot{s}, \quad(0<t<T) .
$$

Let us focus on the properties of $\mathcal{C}(n)$ and $\mathcal{C}_{F}(n)$.

Theorem 2.3. For any positive integer $n$, the set $\mathcal{C}(n)$ is an analytic connected embedded submanifold of $\left(\{\operatorname{Id}\}+C_{0}^{1}\left(\mathbf{R}^{3}\right)^{3}\right) \times\left(C_{0}^{1}\left(\mathbf{R}^{3}\right)^{3}\right)^{n}$ of codimension $N:=3(n+2)(n+1) / 2$.

The definition and the main properties of analytic functions valued in Banach spaces are summarized in the article [19].

Proof. For any $c:=\left(\Theta_{\text {rst }}, \mathcal{V}\right) \in \mathcal{D}_{0}^{1}\left(\mathbf{R}^{3}\right) \times\left(C_{0}^{1}\left(\mathbf{R}^{3}\right)^{3}\right)^{n}$, denote $\mathbf{V}_{0}:=\Theta_{\text {rst }}$ and $\mathcal{V}:=\left(\mathbf{V}_{1}, \ldots, \mathbf{V}_{n}\right)$. Then, define for $k=0,1, \ldots, n$, the functions

$$
\Lambda_{k}: C_{0}^{1}\left(\mathbf{R}^{3}\right)^{3} \times\left(C_{0}^{1}\left(\mathbf{R}^{3}\right)^{3}\right)^{n} \rightarrow \mathbf{R}^{3(n+1-k)}
$$

by

$$
\Lambda_{k}(c):=\left(\int_{\Sigma} \mathbf{V}_{k} \mathrm{~d} x, \int_{\Sigma} \mathbf{V}_{k} \times \mathbf{V}_{k+1} \mathrm{~d} x, \ldots, \int_{\Sigma} \mathbf{V}_{k} \times \mathbf{V}_{n} \mathrm{~d} x\right)^{*} .
$$

Every function $\Lambda_{k}$ is analytic and so is

$$
\Lambda:=\left(\Lambda_{0}, \ldots, \Lambda_{n}\right)^{*}: \mathcal{D}_{0}^{1}\left(\mathbf{R}^{3}\right) \times\left(C_{0}^{1}\left(\mathbf{R}^{3}\right)^{3}\right)^{n} \rightarrow \mathbf{R}^{N}
$$

$(N:=3(n+2)(n+1) / 2)$. In order to prove that $\Lambda^{\prime}(c)$ (the differential of $\Lambda$ at the point $c$ ) is onto for any $c \in \mathcal{C}(n)$, assume that there exist $(n+2)(n+1) / 2$ vectors $\boldsymbol{\alpha}_{i}^{j} \in \mathbf{R}^{3}(0 \leq i \leq j \leq n)$ such that:

$$
\sum_{i=0}^{n} \boldsymbol{\alpha}_{i} \cdot\left\langle\Lambda_{i}^{\prime}(c), c^{h}\right\rangle=\mathbf{0}, \quad \forall c^{h} \in C_{0}^{1}\left(\mathbf{R}^{3}\right)^{3} \times\left(C_{0}^{1}\left(\mathbf{R}^{3}\right)^{3}\right)^{n}
$$

where $\boldsymbol{\alpha}_{i}:=\left(\boldsymbol{\alpha}_{i}^{i}, \boldsymbol{\alpha}_{i}^{i+1}, \ldots, \boldsymbol{\alpha}_{i}^{n}\right)^{*} \in \mathbf{R}^{3(n+1-i)}(i=0, \ldots, n)$ and $c^{h}:=\left(\mathbf{V}_{0}^{h}, \mathcal{V}^{h}\right) \in C_{0}^{1}\left(\mathbf{R}^{3}\right)^{3} \times\left(C_{0}^{1}\left(\mathbf{R}^{3}\right)^{3}\right)^{n}$ with $\mathcal{V}^{h}:=\left(\mathbf{V}_{1}^{h}, \ldots, \mathbf{V}_{n}^{h}\right)$. Reorganizing the terms in $(2.2)$, we obtain that:

$$
\sum_{k=0}^{n} \int_{\Sigma} \mathbf{V}_{k}^{h} \cdot\left[\sum_{j=0}^{k-1} \boldsymbol{\alpha}_{j}^{k} \times \mathbf{V}_{j}+\boldsymbol{\alpha}_{k}^{k}-\sum_{j=k+1}^{n} \boldsymbol{\alpha}_{k}^{j} \times \mathbf{V}_{j}\right] \mathrm{d} x=0
$$

Since this identity has to be satisfied for any $\left(\mathbf{V}_{0}^{h}, \mathcal{V}^{h}\right) \in C_{0}^{1}\left(\mathbf{R}^{3}\right)^{3} \times\left(C_{0}^{1}\left(\mathbf{R}^{3}\right)^{3}\right)^{n}$, we deduce that, for every $k=0, \ldots, n$ :

$$
\sum_{j=0}^{k-1} \boldsymbol{\alpha}_{j}^{k} \times\left.\mathbf{V}_{j}\right|_{\Sigma}+\boldsymbol{\alpha}_{k}^{k}-\sum_{j=k+1}^{n} \boldsymbol{\alpha}_{k}^{j} \times\left.\mathbf{V}_{j}\right|_{\Sigma}=\mathbf{0} .
$$

Integrating this equality over $\Sigma$, we get that $\boldsymbol{\alpha}_{k}^{k}=\mathbf{0}(k=0, \ldots, n)$. Taking into account hypothesis 1 of Definition 2.1, the identity (2.3) with $k=0$ leads to $\boldsymbol{\alpha}_{0}^{j}=\mathbf{0}$ for every $j=1, \ldots, n$. There are no more terms involving $\mathbf{V}_{0}$ in the other equations and invoking again hypothesis 1 we eventually get $\boldsymbol{\alpha}_{i}^{j}=\mathbf{0}$ for $1 \leq i<j \leq n$. So, equality (2.2) entails that $\boldsymbol{\alpha}_{i}=\mathbf{0}$ for all $i=0, \ldots, n$ and the mapping $\Lambda^{\prime}(c)$ is indeed onto for all $c \in \mathcal{C}(n)$.

The linear space $X=\operatorname{Ker} \Lambda^{\prime}(c)$ is closed since $\Lambda$ is analytic. Let $Y$ be an algebraic supplement of $X$ in $C_{0}^{1}\left(\mathbf{R}^{3}\right)^{3} \times\left(C_{0}^{1}\left(\mathbf{R}^{3}\right)^{3}\right)^{n}$, and denote by $P_{Y}$ the linear projection onto $Y$ along $X$. A crucial observation is that 
the linear space $Y$ is isomorphic to $\mathbf{R}^{N}$ and hence it is finite dimensional and closed in $C_{0}^{1}\left(\mathbf{R}^{3}\right)^{3} \times\left(C_{0}^{1}\left(\mathbf{R}^{3}\right)^{3}\right)^{n}$. Define the analytic mapping

$$
\begin{aligned}
f: X \times Y & \rightarrow \mathbf{R}^{N} \\
(x, y) & \mapsto \Lambda(c+x+y) .
\end{aligned}
$$

The mapping $\partial_{y} f(0,0)=\Lambda^{\prime}(c) \circ P_{Y}$ being onto, the implicit function theorem (analytic version in Banach spaces, see [19]) asserts that there exist an open neighborhood $\mathcal{O}_{1}$ of 0 in $X$, an open neighborhood $\mathcal{O}_{2}$ of 0 in $Y$, and an analytic mapping $g: \mathcal{O}_{1} \rightarrow Y$ such that $g(0)=0$ and, for every $(x, y)$ in $\mathcal{O}_{1} \times \mathcal{O}_{2}$, the two following assertions are equivalent:

1. $f(x, y)=0$ (or, in other words, $c+x+y$ belongs to $\mathcal{C}(n)$ );

2. $y=g(x)$.

The analytic mapping $g$ provides a local parameterization of $\mathcal{C}(n)$ in a neighborhood of $c$.

In order to prove that $\mathcal{C}(n)$ is path-connected, consider two elements $c^{\dagger}:=\left(\Theta_{\text {rst }}^{\dagger}, \mathcal{V}^{\dagger}\right)$ and $c^{\ddagger}:=\left(\Theta_{\text {rst }}^{\ddagger}, \mathcal{V}^{\ddagger}\right)$ of $\mathcal{C}(n)$ and denote $\mathcal{V}^{\dagger}:=\left(\mathbf{V}_{1}^{\dagger}, \ldots, \mathbf{V}_{n}^{\dagger}\right)$ and $\mathcal{V}^{\ddagger}:=\left(\mathbf{V}_{1}^{\ddagger}, \ldots, \mathbf{V}_{n}^{\ddagger}\right)$.

According to Definition A.2 (in Appendix A), $\mathcal{D}_{0}^{1}\left(\mathbf{R}^{3}\right)$ is open and connected. This entails that it is always possible to find a continuous, piecewise linear path

$$
t:[0,1] \mapsto \bar{\Theta}_{t} \in \mathcal{D}_{0}^{1}\left(\mathbf{R}^{3}\right)
$$

such that $\bar{\Theta}_{t=0}=\Theta_{\mathrm{rst}}^{\dagger}$ and $\bar{\Theta}_{t=1}=\Theta_{\mathrm{rst}}^{\ddagger}$. We introduce

$$
0=t_{0}<t_{1}<\ldots<t_{k}=1
$$

a subdivision of the interval $[0,1]$ such that $t \mapsto \bar{\Theta}_{t}$ is linear on every subinterval $\left[t_{j}, t_{j+1}\right](j=0, \ldots, k-1)$ and we denote $\bar{\Theta}^{j}:=\bar{\Theta}_{t=t_{j}}(j=0, \ldots, k)$.

Since $C_{0}^{1}\left(\mathbf{R}^{3}\right)^{3}$ is an infinite dimensional Banach space, it is always possible to find by induction $\mathbf{W}_{1}, \mathbf{W}_{2}, \ldots, \mathbf{W}_{n}$ in $C_{0}^{1}\left(\mathbf{R}^{3}\right)^{3}$ such that

1. both families

$$
\left\{\left.\mathbf{W}_{1}\right|_{\Sigma} \cdot \mathbf{e}_{j}, \ldots,\left.\mathbf{W}_{n}\right|_{\Sigma} \cdot \mathbf{e}_{j},\left.\mathbf{V}_{1}^{\dagger}\right|_{\Sigma} \cdot \mathbf{e}_{j}, \ldots,\left.\mathbf{V}_{n}^{\dagger}\right|_{\Sigma} \cdot \mathbf{e}_{j}, j=1,2,3\right\}
$$

and

$$
\left\{\left.\mathbf{W}_{1}\right|_{\Sigma} \cdot \mathbf{e}_{j}, \ldots,\left.\mathbf{W}_{n}\right|_{\Sigma} \cdot \mathbf{e}_{j},\left.\mathbf{V}_{1}^{\ddagger}\right|_{\Sigma} \cdot \mathbf{e}_{j}, \ldots,\left.\mathbf{V}_{n}^{\ddagger}\right|_{\Sigma} \cdot \mathbf{e}_{j}, j=1,2,3\right\}
$$

are free in $C_{0}^{1}\left(\mathbf{R}^{3}\right)$;

2. for any pair of elements $\mathbf{V}, \mathbf{V}^{\prime}$, both picked in the same family, $\int_{\Sigma} \mathbf{V} \mathrm{d} x=\mathbf{0}, \int_{\Sigma} \bar{\Theta}^{j} \times \mathbf{V} \mathrm{d} x=\mathbf{0}$ (for all $j=1, \ldots, k)$ and $\int_{\Sigma} \mathbf{V} \times \mathbf{V}^{\prime} \mathrm{d} x=\mathbf{0}$.

Define now the function

by

$$
t \in[0,1] \mapsto \mathbf{V}_{t}^{i} \in C_{0}^{1}\left(\mathbf{R}^{3}\right)^{3}
$$

$$
\mathbf{V}_{t}^{i}:= \begin{cases}(1-2 t) \mathbf{V}_{i}^{\dagger}+2 t \mathbf{W}_{i} & \text { if } 0 \leq t \leq 1 / 2 \\ (2-2 t) \mathbf{W}_{i}+(2 t-1) \mathbf{V}^{\ddagger} & \text { if } 1 / 2<t \leq 1,\end{cases}
$$

and denote $\mathcal{V}_{t}:=\left(\mathbf{V}_{t}^{1}, \ldots, \mathbf{V}_{t}^{n}\right) \in\left(C_{0}^{1}\left(\mathbf{R}^{3}\right)^{3}\right)^{n}$. Eventually, a continuous function linking $c^{\dagger}$ to $c^{\ddagger}$ is given by $t \in[0,1] \mapsto c_{t} \in \mathcal{C}(n)$ with

$$
c_{t}:= \begin{cases}\left(\Theta_{\mathrm{rst}}^{\dagger}, \mathcal{V}_{3 t / 2}\right) & \text { if } 0 \leq t \leq 1 / 3 \\ \left(\bar{\Theta}_{3 t-1}, \mathcal{V}_{1 / 2}\right) & \text { if } 1 / 3<t \leq 2 / 3 \\ \left(\Theta_{\mathrm{rst}}^{\ddagger}, \mathcal{V}_{3 t / 2-1 / 2}\right) & \text { if } 2 / 3<t \leq 1\end{cases}
$$

We omit the proof of the following corollary, similar to that of the theorem above:

Corollary 2.4. For any positive integer $n$, the set $\mathcal{C}_{F}(n)$ is an analytic connected embedded submanifold of $C_{0}^{1}\left(\mathbf{R}^{3}\right)^{3} \times\left(C_{0}^{1}\left(\mathbf{R}^{3}\right)^{3}\right)^{n} \times \mathbf{R}^{n}$ of codimension $N:=3(n+2)(n+1) / 2$. 
We denote by $\Pi$ the projection of $\mathcal{C}(n)$ onto $\mathcal{D}_{0}^{1}\left(\mathbf{R}^{3}\right)$ defined by $\Pi(c)=\Theta_{\text {rst }}$ for all $c:=\left(\Theta_{\text {rst }}, \mathcal{V}\right) \in \mathcal{C}(n)$. The proof of the following corollary is a straightforward consequence of arguments already used in the proof of Theorem 2.3:

Corollary 2.5. For any positive integer $n$ and for any $\Theta_{\mathrm{rst}} \in \Pi(\mathcal{C}(n))$, the section $\Pi^{-1}\left(\left\{\Theta_{\mathrm{rst}}\right\}\right)$ is an embedded connected analytic submanifold of $\left\{\Theta_{\mathrm{rst}}\right\} \times\left(C_{0}^{1}\left(\mathbf{R}^{3}\right)^{3}\right)^{n}$ (identified with $\left.\left(C_{0}^{1}\left(\mathbf{R}^{3}\right)^{3}\right)^{n}\right)$ of codimension $3 n(n+3) / 2$.

\section{Sensitivity analysis of the matrices $\mathbb{M}(\mathbf{c})$ AND $\mathbb{N}(\mathbf{c})$}

For any positive integers $k$ and $l$, we denote $\mathrm{M}(k, l)$ the vector space of the matrices of size $k \times l$ (or simply $\mathrm{M}(k)$ when $l=k)$.

Theorem 3.1. For any positive integer $n$, the mappings

$$
\mathbf{c} \in \mathcal{C}_{F}(n) \mapsto \mathbb{M}(\mathbf{c}) \in \mathrm{M}(6)
$$

and

$$
\mathbf{c} \in \mathcal{C}_{F}(n) \mapsto \mathbb{N}(\mathbf{c}) \in \mathrm{M}(6, n)
$$

are analytic.

Let us begin with a preliminary lemma, the statement of which requires introducing some material.

Thus, we denote $F:=\mathbf{R}^{3} \backslash \bar{B}$ (remember that $B$ is the unit ball, $\Sigma:=\partial B$ and $\mathbf{n}$ is the unit normal to $\Sigma$ directed toward the interior of $B)$. For all $\Xi \in \mathcal{D}_{0}^{1}\left(\mathbf{R}^{3}\right)$, we set $\mathcal{B}_{\Xi}:=\Xi(B), \mathcal{F}_{\Xi}:=\Xi(F)$ and $\Sigma_{\Xi}:=\Xi(\Sigma)$. We denote $\mathbf{q}:=(\Xi, \mathcal{W})$, with $\mathcal{W}:=\left(\mathbf{W}^{1}, \mathbf{W}^{2}\right) \subset\left(C_{0}^{1}\left(\mathbf{R}^{3}\right)^{3}\right)^{2}$, the elements of $\mathcal{Q}:=\mathcal{D}_{0}^{1}\left(\mathbf{R}^{3}\right) \times\left(C_{0}^{1}\left(\mathbf{R}^{3}\right)^{3}\right)^{2}$ and $\mathbf{w}_{\Xi}^{i}:=\mathbf{W}^{i}\left(\Xi^{-1}\right)(i=1,2)$. Finally, for every $\mathbf{q} \in \mathcal{Q}$, we define:

$$
\Phi(\mathbf{q}):=\int_{\mathcal{F}_{\Xi}} D\left(\mathbf{u}_{\mathbf{q}}^{1}\right): D\left(\mathbf{u}_{\mathbf{q}}^{2}\right) \mathrm{d} x
$$

where, for every $i=1,2$, there exists a function $p_{\mathbf{q}}^{i} \in L^{2}\left(\mathcal{F}_{\Xi}\right)$ such that the pair $\left(\mathbf{u}_{\mathbf{q}}^{i}, p_{\mathbf{q}}^{i}\right) \in\left(W_{0}^{1}\left(\mathcal{F}_{\Xi}\right)\right)^{3} \times L^{2}\left(\mathcal{F}_{\Xi}\right)$ solves the Stokes system:

$$
\begin{aligned}
& -\Delta \mathbf{u}_{\mathbf{q}}^{i}+\nabla p_{\mathbf{q}}^{i}=\mathbf{0} \quad \text { in } \mathcal{F}_{\Xi}, \\
& \nabla \cdot \mathbf{u}_{\mathbf{q}}^{i}=0 \quad \text { in } \mathcal{F}_{\Xi}, \\
& \mathbf{u}_{\mathbf{q}}^{i}=\mathbf{w}_{\Xi}^{i} \quad \text { on } \Sigma_{\Xi} \text {. }
\end{aligned}
$$

The first equation has to be understood in the weak sense, namely:

$$
\int_{\mathcal{F}_{\Xi}} \nabla \mathbf{u}_{\mathbf{q}}^{i}: \nabla \mathbf{v} \mathrm{d} x-\int_{\Sigma_{\Xi}} p_{\mathbf{q}}^{i}(\nabla \cdot \mathbf{v}) \mathrm{d} x=\mathbf{0}, \quad \forall \mathbf{v} \in\left(\stackrel{\circ}{W_{0}^{1}}\left(\mathcal{F}_{\Xi}\right)\right)^{3} .
$$

Recall that the function spaces are defined in the Appendix A.

Lemma 3.2. The mapping $\mathbf{q} \in \mathcal{Q} \mapsto \Phi(\mathbf{q}) \in \mathbf{R}$ is analytic.

Proof. We pull back equality (3.3) onto the domain $F$ using the diffeomorphism $\Xi$. We get:

$$
\int_{F} \nabla \mathbf{U}_{\mathbf{q}}^{i} \mathbb{A}_{\Xi}: \nabla \mathbf{V} \mathrm{d} x-\int_{F} P_{\mathbf{q}}^{i} \mathbb{B} \Xi: \nabla \mathbf{V} \mathrm{d} x=\mathbf{0}, \quad \forall \mathbf{V} \in\left(\stackrel{\circ}{W_{0}^{1}}(F)\right)^{3},
$$

where $\mathbf{U}_{\mathbf{q}}^{i}:=\mathbf{u}_{\mathbf{q}}^{i} \circ \Xi, P_{\mathbf{q}}^{i}:=p_{\mathbf{q}}^{i} \circ \Xi, J_{\Xi}:=\operatorname{det}(\nabla \Xi), \mathbb{A}_{\Xi}:=\left(\nabla \Xi^{*} \nabla \Xi\right)^{-1} J_{\Xi}$ and $\mathbb{B}_{\Xi}:=\left(\nabla \Xi^{*}\right)^{-1} J_{\Xi}$. 
Likewise, $(3.2 \mathrm{~b}-3.2 \mathrm{c})$ can be turned into:

$$
\begin{aligned}
\mathbb{B}_{\Xi}: \nabla \mathbf{U}_{\mathbf{q}}^{i} & =0, & & \text { in } F, \\
\mathbf{U}_{\mathbf{q}}^{i} & =\mathbf{W}^{i} & & \text { on } \Sigma .
\end{aligned}
$$

We now claim that the mapping

$$
\Xi \in \mathcal{D}_{0}^{1}\left(\mathbf{R}^{3}\right) \mapsto \mathbb{A}_{\Xi} \in E_{0}^{0}\left(\mathbf{R}^{3}, \mathrm{M}(3)\right)
$$

is analytic. Indeed, the mappings

$$
\begin{aligned}
\Xi \in \mathcal{D}_{0}^{1}\left(\mathbf{R}^{3}\right) & \mapsto \nabla \Xi^{*} \nabla \Xi \in E_{0}^{0}\left(\mathbf{R}^{3}, \mathrm{M}(3)\right) \\
A \in E_{0}^{0}\left(\mathbf{R}^{3}, \mathrm{M}(3)\right) & \mapsto A^{-1} \in E_{0}^{0}\left(\mathbf{R}^{3}, \mathrm{M}(3)\right) \\
\Xi \in \mathcal{D}_{0}^{1}\left(\mathbf{R}^{3}\right) & \mapsto J_{\Xi} \in C_{0}^{0}\left(\mathbf{R}^{3}\right)
\end{aligned}
$$

are analytic. Then, for $i=1,2$, we define the analytic functions

$$
\Gamma^{i}: \mathcal{Q} \times\left(W_{0}^{1}(F)\right)^{3} \times L^{2}(F) \rightarrow\left(W_{0}^{-1}(F)\right)^{3} \times L^{2}(F) \times\left(H^{1 / 2}(\Sigma)\right)^{3}
$$

by:

$$
\Gamma^{i}(\mathbf{q}, \mathbf{U}, P):=\left(\begin{array}{c}
\left\langle\mathbb{A}_{\Xi}, \mathbf{U}_{,} \cdot\right\rangle-\left\langle\mathbb{B}_{\Xi}, P, \cdot\right\rangle \\
\mathbb{B}_{\Xi}: \nabla \mathbf{U} \\
\gamma_{\Sigma}\left(\mathbf{U}-\mathbf{W}^{i}\right)
\end{array}\right)
$$

where $\gamma_{\Sigma}:\left(W^{1}(F)\right)^{3} \rightarrow\left(H^{1 / 2}(\Sigma)\right)^{3}$ is the trace operator and

$$
\begin{aligned}
\left\langle\mathbb{A}_{\Xi}, \mathbf{U}, \mathbf{V}\right\rangle:=\int_{\mathcal{F}} \nabla \mathbf{U A}_{\Xi}: \nabla \mathbf{V} \mathrm{d} x, \quad\left(\mathbf{U} \in\left(W^{1}(F)\right)^{3}, \mathbf{V} \in\left(W_{0}^{1}(F)\right)^{3}\right), \\
\left\langle\mathbb{B}_{\Xi}, P, \mathbf{V}\right\rangle:=\int_{\mathcal{F}} P \mathbb{B}_{\Xi}: \nabla \mathbf{V} \mathrm{d} x, \quad\left(P \in L^{2}(F), \mathbf{V} \in\left(W_{0}^{1}(F)\right)^{3}\right) .
\end{aligned}
$$

We wish now to apply the implicit function theorem (analytic version in Banach spaces, as stated in [19]) to the analytic function $\Gamma^{i}$. Observe however that we are only interested in the regularity result. Indeed, according to Proposition D.5, we already know that for all $i=1,2$ and all $\mathbf{q} \in \mathcal{Q}$, there exists a unique pair $\left(U_{\mathbf{q}}^{i}, P_{\mathbf{q}}^{i}\right) \in\left(W_{0}^{1}(F)\right)^{3} \times L^{2}(F)$ such that $\Gamma^{i}\left(\mathbf{q}, \mathbf{U}_{\mathbf{q}}^{i}, P_{\mathbf{q}}^{i}\right)=\mathbf{0}$.

For every $\mathbf{q} \in \mathcal{Q}$, the partial derivative $\partial_{(U, P)} \Gamma^{i}\left(\mathbf{q}, U_{\mathbf{q}}^{i}, P_{\mathbf{q}}^{i}\right)$ can be readily computed. Indeed, we have:

$$
\left\langle\partial_{(U, P)} \Gamma^{i}\left(\mathbf{q}, U_{\mathbf{q}}^{i}, P_{\mathbf{q}}^{i}\right),(\chi, \pi)\right\rangle=\left(\begin{array}{c}
\left\langle\mathbb{A}_{\Xi}, \boldsymbol{\chi}, \cdot\right\rangle-\left\langle\mathbb{B}_{\Xi}, \pi, \cdot\right\rangle \\
\mathbb{B}_{\Xi}: \nabla \chi \\
\gamma_{\Sigma}(\boldsymbol{\chi})
\end{array}\right), \quad \forall(\boldsymbol{\chi}, \pi) \in\left(W_{0}^{1}(F)\right)^{3} \times L^{2}(\Sigma) .
$$

Let $(\mathbf{f}, \eta, \mathbf{g})$ be any element of $\left(W_{0}^{-1}(F)\right)^{3} \times L^{2}(F) \times\left(H^{1 / 2}(F)\right)^{3}$. The equation

$$
\left\langle\partial_{(U, P)} \Gamma^{i}\left(\mathbf{q},\left(U_{\mathbf{q}}^{i}, P_{\mathbf{q}}^{i}\right),(\chi, \pi)\right\rangle=(\mathbf{f}, \eta, \mathbf{g}),\right.
$$

is equivalent to:

$$
\begin{aligned}
\int_{F} \nabla \chi \mathbb{A}_{\Xi}: \nabla \mathbf{V} \mathrm{d} x-\int_{F} \pi \mathbb{B}_{\Xi}: \nabla \mathbf{V} \mathrm{d} x & =\langle\mathbf{f}, \mathbf{V}\rangle_{\left(W_{0}^{-1}(F)\right)^{3} \times\left(W_{0}^{1}(F)\right)^{3}}, \quad \forall \mathbf{V} \in\left(\stackrel{\circ}{\left.W_{0}^{1}(F)\right)^{3},}\right. \\
\mathbb{B}_{\Xi}: \nabla \chi & =\eta, \quad \text { in } F, \\
\chi & =\mathbf{g} \quad \text { on } \Sigma .
\end{aligned}
$$


According to Proposition D.5, there exists a unique solution

$$
(\chi, \pi) \in\left(W_{0}^{1}(F)\right)^{3} \times L^{2}(F)
$$

such that

$$
\|\chi\|_{\left(W_{0}^{1}(F)\right)^{3}}+\|\pi\|_{L^{2}(F)} \leq C_{\Xi}\left[\|\mathbf{f}\|_{\left(W_{0}^{-1}\right)^{3}}+\|\eta\|_{L^{2}(F)}+\|\mathbf{g}\|_{\left(H^{1 / 2}(\Sigma)\right)^{3}}\right]
$$

where the constant $C_{\Xi}>0$ depends on $\Xi$ only. We infer that for every $\mathbf{q} \in \mathcal{Q}, \partial_{(U, P)} \Gamma^{i}\left(\mathbf{q}, U_{\mathbf{q}}^{i}, P_{\mathbf{q}}^{i}\right)$ is a continuous isomorphism from $\left(W_{0}^{1}(F)\right)^{3} \times L^{2}(F)$ onto $\left(W_{0}^{-1}(F)\right)^{3} \times L^{2}(F) \times\left(H^{1 / 2}(\Sigma)\right)^{3}$. The implicit function theorem applies and asserts that the mapping

$$
\mathbf{q} \in \mathcal{Q} \mapsto\left(\mathbf{U}_{\mathbf{q}}^{i}, P_{\mathbf{q}}^{i}\right) \in\left(W_{0}^{1}(F)\right)^{3} \times L^{2}(F) \quad(i=1,2)
$$

is analytic.

To conclude the proof, it remains only to observe that the function $\Phi(\mathbf{q})$ introduced in (3.1) can be rewritten, upon a change of variables as

$$
\Phi(\mathbf{q})=\frac{1}{4} \int_{F}\left(\nabla U_{\mathbf{q}}^{1} \nabla \Xi^{-1}+\left(\nabla U_{\mathbf{q}}^{1} \nabla \Xi^{-1}\right)^{*}\right):\left(\nabla U_{\mathbf{q}}^{2} \nabla \Xi^{-1}+\left(\nabla U_{\mathbf{q}}^{2} \nabla \Xi^{-1}\right)^{*}\right) J_{\Xi} \mathrm{d} x,
$$

which is analytic as a composition of analytic functions.

We can now give the proof of Theorem 3.1.

Proof. For any $\mathbf{c}:=(c, s) \in \mathcal{C}_{F}(n)$, where $c:=\left(\Theta_{\text {rst }}, \mathcal{V}\right)$, we apply the lemma with

$$
\Xi:=\Theta_{\mathrm{rst}}+\sum_{i=1}^{n} s_{i} \mathbf{V}_{i} \quad \text { and } \quad \mathbf{W}^{1}, \mathbf{W}^{2} \in\left\{\mathbf{e}_{i} \times \Xi, \mathbf{e}_{i}, i=1,2,3\right\}
$$

to get that the mapping $\mathbf{c} \in \mathcal{C}_{F}(n) \mapsto \mathbb{M}(\mathbf{c}) \in \mathrm{M}(6)$ is analytic.

To prove the analyticity of the elements of $\mathbb{N}(\mathbf{c})$, we apply the lemma again with

$$
\Xi:=\Theta_{\mathrm{rst}}+\sum_{i=1}^{n} s_{i} \mathbf{V}_{i}, \quad \mathbf{W}^{1} \in\left\{\mathbf{e}_{i} \times \Xi, \mathbf{e}_{i}, i=1,2,3\right\} \text { and } \mathbf{W}^{2} \in\left\{\mathbf{V}_{1}, \ldots, \mathbf{V}_{n}\right\}
$$

\section{Control problem}

\subsection{Controllable swimmer signature}

Let us fix $c \in \mathcal{C}(n)$ (for some positive integer $n$ ) and recall that $\mathcal{S}(c)$ is the connected open subspace of $\mathbf{R}^{n}$ such that $(c, s) \in \mathcal{C}_{F}(n)$. Introducing $\left(\mathbf{f}_{1}, \ldots, \mathbf{f}_{n}\right)$ an ordered orthonormal basis of $\mathbf{R}^{n}$, we can seek the function

$$
t \in[0, T] \mapsto s(t) \in \mathcal{S}(c)
$$

as the solution of the ODE

$$
\dot{s}(t)=\sum_{i=1}^{n} \lambda_{i}(t) \mathbf{f}_{i}
$$

where the functions

$$
\lambda_{i}: t \in[0, T] \mapsto \lambda_{i}(t) \in \mathbf{R}
$$

are the new controls, and rewrite once more the dynamics (2.1) as:

$$
\left(\begin{array}{c}
\Omega \\
\mathbf{v} \\
\dot{s}
\end{array}\right)=\sum_{i=1}^{n} \lambda_{i}(t)\left(\begin{array}{c}
-\mathbb{M}(c, s)^{-1} \mathbb{N}(c, s) \mathbf{f}_{i} \\
\mathbf{f}_{i}
\end{array}\right), \quad(0<t<T) .
$$


It is worth remarking that in this form, $s$ is no more the control but a state variable and $c \in \mathcal{C}(n)$ is a parameter of the dynamics. Considering (4.1), we are quite naturally led to introduce, for all $\mathbf{c} \in \mathcal{C}_{F}(n)$, the vector fields

$$
\begin{aligned}
& \mathbf{X}_{i}(\mathbf{c}):=-\mathbb{M}(\mathbf{c})^{-1} \mathbb{N}(\mathbf{c}) \mathbf{f}_{i} \in \mathbf{R}^{6} \\
& \mathbf{Y}_{i}(\mathbf{c}):=\left(\hat{\mathbf{X}}_{i}^{1}(\mathbf{c}), \mathbf{X}_{i}^{2}(\mathbf{c}), \mathbf{f}_{i}\right)^{*} \in T_{\mathrm{Id}} \mathrm{SO}(3) \times \mathbf{R}^{3} \times \mathbf{R}^{n}
\end{aligned}
$$

(we have used here the notation $\mathbf{X}_{i}:=\left(\mathbf{X}_{i}^{1}, \mathbf{X}_{i}^{2}\right)^{*} \in \mathbf{R}^{3} \times \mathbf{R}^{3}$ ) and

$$
\mathbf{Z}_{c}^{i}(R, s):=\mathcal{R}_{R} \mathbf{Y}_{i}(\mathbf{c}) \in T_{R} \mathrm{SO}(3) \times \mathbf{R}^{3} \times \mathbf{R}^{n}
$$

where $\mathcal{R}_{R}:=\operatorname{diag}(R, R, \mathrm{Id}) \in \mathrm{SO}(6+n)$ is a bloc diagonal matrix. The dynamics (4.1) and the ODE (1.7b) can be gathered into a unique differential system:

$$
\frac{\mathrm{d}}{\mathrm{d} t}\left(\begin{array}{c}
R \\
\mathbf{r} \\
s
\end{array}\right)=\sum_{i=1}^{n} \lambda_{i}(t) \mathbf{Z}_{c}^{i}(R, s), \quad(0<t<T) .
$$

For every $i=1, \ldots, n$, the function

$$
(R, \mathbf{r}, s) \in \mathrm{SO}(3) \times \mathbf{R}^{3} \times \mathcal{S}(c) \mapsto \mathbf{Z}_{c}^{i}(R, s) \in T_{R} \mathrm{SO}(3) \times \mathbf{R}^{3} \times \mathbf{R}^{n}
$$

can be seen as an analytic vector field (constant in $\mathbf{r}$ ) on the analytic connected manifold

$$
\mathcal{M}(c):=\mathrm{SO}(3) \times \mathbf{R}^{3} \times \mathcal{S}(c) .
$$

We denote $\zeta$ any element $(R, \mathbf{r}, s) \in \mathcal{M}(c)$ and we define $\mathcal{Z}(c)$ as the family of vector fields $\left(\mathbf{Z}_{c}^{i}\right)_{1 \leq i \leq n}$ on $\mathcal{M}(c)$.

Lemma 4.1. Let $c$ be a $S S$ fixed in $\mathcal{C}(n)$ (n a positive integer). If there exists $\zeta \in \mathcal{M}(c)$ such that

$$
\operatorname{dim} \operatorname{Lie}_{\zeta} \mathcal{Z}(c)=6+n,
$$

then the orbit of $\mathcal{Z}(c)$ through any $\zeta \in \mathcal{M}(c)$ is equal to the whole manifold $\mathcal{M}(c)$.

Proof. Rashevsky Chow Theorem (see [1]) applies: If $\operatorname{Lie}_{\zeta} \mathcal{Z}(c)=T_{\zeta} \mathcal{M}(c)$ for all $\zeta \in \mathcal{M}(c)$ (or more precisely, for all $(R, s) \in \mathrm{SO}(3) \times \mathcal{S}(c)$ since $\mathbf{Z}_{c}^{i}$ does not depend on $\left.\mathbf{r}\right)$ then the orbit of $\mathcal{Z}(c)$ through any point of $\mathcal{M}(c)$ is equal to the whole manifold.

Let us compute the Lie bracket $\left[\mathbf{Z}_{c}^{i}(R, s), \mathbf{Z}_{c}^{j}(R, s)\right]$ for $1 \leq i, j \leq n$ and $(R, s) \in \mathrm{SO}(3) \times \mathcal{S}(c)$. We get:

$$
\left[\mathbf{Z}_{c}^{i}(R, s), \mathbf{Z}_{c}^{j}(R, s)\right]=\mathcal{R}_{R}\left(\begin{array}{c}
\left(\mathbf{X}_{i}^{1 \times \mathbf{X}_{j}^{1}}\right)(\mathbf{c}) \\
\left(\mathbf{X}_{i}^{1} \times \mathbf{X}_{j}^{2}-\mathbf{X}_{j}^{1} \times \mathbf{X}_{i}^{2}\right)(\mathbf{c}) \\
\mathbf{0}
\end{array}\right)+\mathcal{R}_{R}\left(\begin{array}{c}
\left(\partial_{s_{i}} \mathbf{X}_{j}^{1}-\partial_{s_{j}} \mathbf{X}_{i}^{1}\right)(\mathbf{c}) \\
\left(\partial_{s_{i}} \mathbf{X}_{j}^{2}-\partial_{s_{j}} \mathbf{X}_{i}^{2}\right)(\mathbf{c}) \\
\mathbf{0}
\end{array}\right)
$$

By induction, we can similarly prove that the Lie brackets of any order at any point $\zeta \in \mathcal{M}(c)$ have the same general form, namely the matrix $\mathcal{R}_{R}$ multiplied by an element of $T_{(\mathrm{Id}, \mathbf{0}, s)} \mathcal{M}(c)$. We deduce that the dimension of the Lie algebra at any point of $\mathcal{M}(c)$ depends only on $s$. According to the Orbit Theorem (see [1]), the dimension of the Lie algebra is constant along any orbit. But according to the particular form of the vector fields $\mathbf{Z}_{c}^{i}$ (whose last $n$ components form a basis of $\mathbf{R}^{n}$ ), the projection of any orbit on $\mathcal{S}(c)$ turns out to be the whole set $\mathcal{S}(c)$ (or, in other words, for any $s \in \mathcal{S}(c)$ and for any orbit, there is a point of the orbit for which the last component is $s$ ).

Assume now that $\operatorname{dim} \operatorname{Lie}_{\zeta^{*}} \mathcal{Z}(c)=6+n$ at some particular point $\zeta^{*}:=\left(R^{*}, \mathbf{r}^{*}, s^{*}\right) \in \mathcal{M}(c)$. Then, according to the Orbit Theorem, for any $s \in \mathcal{S}(c)$, there exists at least one point $\left(R_{s}, \mathbf{r}_{s}, s\right) \in \mathcal{M}(c)$ such that

$$
\operatorname{dim} \operatorname{Lie}_{\left(R_{s}, \mathbf{r}_{s}, s\right)} \mathcal{Z}(c)=6+n .
$$

But since the dimension of the Lie algebra does not depend on the variables $R$ and $\mathbf{r}$, we conclude that $\operatorname{dim} \operatorname{Lie}_{\zeta} \mathcal{Z}(c)=6+n$ for all $\zeta \in \mathcal{M}(c)$. 
Definition 4.2. We say that $c$, a $\mathrm{SS}$ in $\mathcal{C}(n)$ (for some integer $n$ ) is controllable if there exists $\zeta \in \mathcal{M}(c)$ such that $\operatorname{dim}_{\operatorname{Lie}} \mathcal{Z}(c)=6+n$.

It is obvious that for a SS to be controllable, the integer $n$ has to be larger or equal to 2 . The following result is quite classical (a proof can be found in [5]):

Proposition 4.3. Let $c \in \mathcal{C}(n)$ (for some integer $n$ ) be controllable (with the usual notation $c:=\left(\Theta_{\text {rst }}, \mathcal{V}\right.$ ), $\mathcal{V}:=\left(\mathbf{V}_{1}, \ldots, \mathbf{V}_{n}\right)$ and $\Theta_{s}:=\Theta_{\mathrm{rst}}+\sum_{i=1}^{n} s_{i} \mathbf{V}_{i}$ for every $\left.s \in \mathcal{S}(c)\right)$.

Then for any given continuous function

$$
t \in[0, T] \mapsto(\bar{R}(t), \overline{\mathbf{r}}(t), \bar{s}(t)) \in \mathrm{SO}(3) \times \mathbf{R}^{3} \times \mathcal{S}(c)
$$

and for any $\varepsilon>0$, there exist $n C^{1}$ functions $\lambda_{i}:[0, T] \rightarrow \mathbf{R}(i=1, \ldots, n)$ such that:

1. $\sup _{t \in[0, T]}\left(\|\bar{R}(t)-R(t)\|_{\mathrm{M}(3)}+\|\overline{\mathbf{r}}(t)-\mathbf{r}(t)\|_{\mathbf{R}^{3}}+\left\|\Theta_{\bar{s}(t)}-\Theta_{s(t)}\right\|_{C_{0}^{1}\left(\mathbf{R}^{3}\right)^{3}}\right)<\varepsilon ;$

2. $R(T)=\bar{R}(T), \mathbf{r}(T)=\overline{\mathbf{r}}(T)$ and $s(T)=\bar{s}(T)$;

where $t \in[0, T] \mapsto(R(t), \mathbf{r}(t), s(t)) \in \mathcal{M}(c)$ is the unique solution to the ODE (4.2) with Cauchy data $R(0)=\bar{R}(0) \in \mathrm{SO}(3), \mathbf{r}(0)=\overline{\mathbf{r}}(0) \in \mathbf{R}^{3}, s(0)=\bar{s}(0) \in \mathcal{S}(c)$.

Let us mention some other quite elementary properties that will be used later on:

\section{Proposition 4.4.}

1. If $c:=\left(\Theta_{\mathrm{rst}}, \mathcal{V}\right) \in \mathcal{C}(n)(n \geq 2)$ is a controllable $S S$ with $\mathcal{V}:=\left(\mathbf{V}_{1}, \ldots, \mathbf{V}_{n}\right) \in\left(C_{0}^{1}\left(\mathbf{R}^{3}\right)^{3}\right)^{n}$ then any $c^{+}:=\left(\Theta_{\mathrm{rst}}, \mathcal{V}^{+}\right) \in \mathcal{C}(n+1)$ such that

$$
\mathcal{V}^{+}:=\left(\mathbf{V}_{1}, \ldots, \mathbf{V}_{n}, \mathbf{V}_{n+1}\right) \in\left(C_{0}^{1}\left(\mathbf{R}^{3}\right)^{3}\right)^{n+1}
$$

(for some $\mathbf{V}_{n+1} \in C_{0}^{m}\left(\mathbf{R}^{3}\right)^{3}$ ) is a controllable $S S$ as well.

2. If $c:=\left(\Theta_{\mathrm{rst}}, \mathcal{V}\right) \in \mathcal{C}(n)(n \geq 2)$ is a controllable $S S$, then for any

$$
\Theta_{\mathrm{rst}}^{\star} \in\left\{\Theta_{\mathrm{rst}}+\sum_{i=1}^{n} s_{i} \mathbf{V}_{i}, s \in \mathcal{S}(c)\right\}
$$

the element $c^{\star}:=\left(\Theta_{\mathrm{rst}}^{\star}, \mathcal{V}\right)$ belongs to $\mathcal{C}(n)$ and is a controllable $S S$ as well.

3. If $c:=\left(\Theta_{\mathrm{rst}}, \mathcal{V}\right) \in \mathcal{C}(n)(n \geq 2)$ is a controllable $S S$, then all of the controllable $S S$ in the section $\Pi^{-1}\left(\left\{\Theta_{\mathrm{rst}}\right\}\right)$ form an open dense subset of $\Pi^{-1}\left(\left\{\Theta_{\mathrm{rst}}\right\}\right)$ (for the induced topology).

4. If there exists a $S S$ in $\mathcal{C}(n)$ for some $n \geq 2$ then, for any $k \geq n$, all of the controllable $S S$ in $\mathcal{C}(k)$ form an open dense subset of $\mathcal{C}(k)$ (for the induced topology).

Proof. The two first assertions are obvious so let us address directly the third point.

Denote $\mathcal{E}_{k}$ ( $k$ positive integer) the set of all of the vectors fields on $\mathcal{M}(c)$ obtained as Lie brackets of order lower or equal to $k$ from elements of $\mathcal{Z}(c)$. Then, consider the determinants of all of the different families of $6+n$ elements of $\mathcal{E}_{k}$ as analytic functions in the variable $\mathcal{V}$ (the other variables $\Theta_{\text {rst }}$ and $s=0$ being fixed). Since $c$ is controllable, there exist at least one $k$ and one family of $6+n$ elements in $\mathcal{E}_{k}$ whose determinant is nonzero. According to Corollary 2.5 and basic properties of analytic functions (see [19]), the determinant can vanish only in a closed subset with empty interior of the section $\Pi^{-1}\left(\left\{\Theta_{\mathrm{rst}}\right\}\right)$ (for the induced topology). The proof of the last point is similar. 


\subsection{Building a controllable swimmer signature}

In this subsection, we are interested in computing the Lie brackets of first order $\left[\mathbf{Z}_{c}^{i}(R, s), \mathbf{Z}_{c}^{j}(R, s)\right]$ at $(R, s)=(\mathrm{Id}, 0)$, for a particular SS $c:=(\mathrm{Id}, \mathcal{V}) \in \mathcal{C}(4)$ (so the shape of the swimmer at rest is the unit ball). We make use of the usual notation $\mathcal{V}:=\left(\mathbf{V}_{1}, \ldots, \mathbf{V}_{4}\right)$ (to be specified later on), $s=\left(s_{1}, \ldots, s_{4}\right) \in \mathcal{S}(c)$ and $\mathbf{c}:=(c, s)$.

To carry out the aforementioned task, we introduce the classical spherical coordinates $(\varrho, \alpha, \beta)$ such that, for all $x:=\left(x_{1}, x_{2}, x_{3}\right)^{*} \in \mathbf{R}^{3}, x \neq 0$, we have

$$
\begin{aligned}
& x_{1}=\varrho \cos (\alpha) \sin (\beta), \\
& x_{2}=\varrho \sin (\alpha) \sin (\beta), \\
& x_{3}=\varrho \cos (\beta) .
\end{aligned}
$$

At each point $(\varrho, \alpha, \beta)$ we define the related local frame $\left(\mathbf{e}_{\varrho}, \mathbf{e}_{\alpha}, \mathbf{e}_{\beta}\right)$. For any $n \geq 1$, we call rigid spherical harmonics of degree $-(n+1)$ any function having the form:

$$
(\varrho, \alpha, \beta) \mapsto \varrho^{-(n+1)} \sum_{m=-n}^{n} \gamma_{m} Y_{n, m}(\cos \beta, \alpha),
$$

where $\gamma_{-n}, \ldots, \gamma_{n} \in \mathbf{R}$ and $Y_{n, m}$ are the classical spherical harmonics of degree $n \in \mathbf{N}$ and order $m \in\{-n, \ldots, n\}$.

According to Lamb, [10] (one can also see the book of Happel and Brenner, [9], Chap. 3.2, p. 62), the solution $(\mathbf{u}, p)$ of the Stokes equations around an immersed body of any shape can be decomposed as follows (in the body frame):

$$
\begin{aligned}
\mathbf{u}=\sum_{n=0}^{+\infty}\left(\nabla \times\left(\chi_{-(n+1)} \varrho \mathbf{e}_{\varrho}\right)+\nabla \phi_{-(n+1)}\right. & -\frac{n-2}{2 n(2 n-1)} \varrho^{2} \nabla p_{-(n+1)} \\
& \left.+\frac{n+1}{n(2 n-1)} p_{-(n+1)} \varrho \mathbf{e}_{\varrho}\right), \\
p=\sum_{n=0}^{+\infty} p_{-(n+1)}, &
\end{aligned}
$$

where $p_{-(n+1)}, \chi_{-(n+1)}$ and $\phi_{-(n+1)}$ are solid spherical harmonics of degree $-(n+1)$.

The functions $p_{-(n+1)}, \chi_{-(n+1)}$ and $\phi_{-(n+1)}$ (or more precisely the coefficients $\gamma_{k}, k \in\{-n, \ldots, n\}$ arising in (4.4)) have to be determined in order to satisfy the boundary conditions on the surface on the body. This can be done following a method given in [3].

\section{Case of a spherical body}

The first step consists in considering a spherical rigid body (of radius $R>0$ ). So, we want to compute the solution of the exterior Stokes problem in the form (4.5) satisfying the boundary condition $\left.\mathbf{u}\right|_{\varrho=R}=\mathbf{v}_{0}$, where $\mathbf{v}_{0}$ is a smooth, given velocity field on the body's boundary.

The leading idea is that, instead of trying to equalize the three components of the velocity on the body's boundary, it is more simple to equalize the normal component of the velocity, the tangential part of the divergence and the normal component of the rotational. 
We recall the general expressions of the gradient, the divergence and the rotational in spherical coordinates:

$$
\begin{aligned}
\nabla \varphi & =\partial_{\varrho} \varphi \mathbf{e}_{\varrho}+\frac{1}{\varrho} \partial_{\alpha} \varphi \mathbf{e}_{\alpha}+\frac{1}{\varrho \sin \alpha} \partial_{\beta} \varphi \mathbf{e}_{\beta} \\
\operatorname{div} \mathbf{u}= & \frac{1}{\varrho^{2}} \partial_{\varrho}\left(\varrho^{2} \mathbf{u}_{\varrho}\right)+\frac{1}{\varrho \sin \alpha} \partial_{\alpha}\left(\sin \alpha \mathbf{u}_{\alpha}\right)+\frac{1}{\varrho \sin \alpha} \partial_{\beta} \mathbf{u}_{\beta}, \\
\nabla \times \mathbf{u} & =\frac{1}{\varrho \sin \alpha}\left(\partial_{\alpha}\left(\sin \alpha \mathbf{u}_{\beta}\right)-\partial_{\beta} \mathbf{u}_{\alpha}\right) \mathbf{e}_{\varrho}+\frac{1}{\varrho}\left(\frac{1}{\sin \alpha} \partial_{\beta} \mathbf{u}_{\varrho}-\partial_{\varrho}\left(\varrho \mathbf{u}_{\beta}\right)\right) \mathbf{e}_{\alpha} \\
& +\frac{1}{\varrho}\left(\partial_{\varrho}\left(\varrho \mathbf{u}_{\alpha}\right)-\partial_{\alpha} \mathbf{u}_{\varrho}\right) \mathbf{e}_{\beta} .
\end{aligned}
$$

We deduce that the expressions of the quantities to be equalized are (besides the normal component $\mathbf{u} \cdot \mathbf{e}_{\varrho}$ of the velocity, which is obvious):

$$
\begin{gathered}
\varrho\left(\nabla\left(\mathbf{u} \cdot \mathbf{e}_{\varrho}\right) \cdot \mathbf{e}_{\varrho}-\operatorname{div} \mathbf{u}\right)=-2 \mathbf{u}_{\varrho}-\frac{1}{\sin \alpha}\left(\partial_{\alpha}\left(\sin \alpha \mathbf{u}_{\alpha}\right)+\partial_{\beta} \mathbf{u}_{\beta}\right) ; \\
\varrho \nabla \times \mathbf{u} \cdot \mathbf{e}_{\varrho}=\frac{1}{\sin \alpha}\left(\partial_{\alpha}\left(\sin \alpha \mathbf{u}_{\beta}\right)-\partial_{\beta} \mathbf{u}_{\alpha}\right) .
\end{gathered}
$$

Sticking to the notation of [3], we decompose these quantities in spherical harmonics:

$$
\begin{aligned}
\mathbf{v}_{0} \cdot \mathbf{e}_{\varrho} & =\sum_{n=0}^{\infty} X_{n}, \\
-2 \mathbf{v}_{0} \cdot \mathbf{e}_{\varrho}-\frac{1}{\sin \alpha}\left(\partial_{\alpha}\left(\sin \alpha \mathbf{v}_{0} \cdot \mathbf{e}_{\alpha}\right)+\partial_{\beta}\left(\mathbf{v}_{0} \cdot \mathbf{e}_{\beta}\right)\right) & =\sum_{n=0}^{\infty} Y_{n} \\
\frac{1}{\sin \alpha}\left(\partial_{\alpha}\left(\sin \alpha \mathbf{v}_{0} \cdot \mathbf{e}_{\beta}\right)-\partial_{\beta}\left(\mathbf{v}_{0} \cdot \mathbf{e}_{\alpha}\right)\right) & =\sum_{n=0}^{\infty} Z_{n},
\end{aligned}
$$

where $X_{n}, Y_{n}$ and $Z_{n}$ stand for spherical harmonics of degree $n$. Using (4.5), we end up with the following system:

$$
\begin{aligned}
& p_{-(n+1)}=\frac{2 n-1}{n+1}\left((n+2) X_{n}+Y_{n}\right), \\
& \phi_{-(n+1)}=\frac{1}{2(n+1)}\left(n X_{n}+Y_{n}\right), \\
& \chi_{-(n+1)}= \begin{cases}0 & n=0, \\
\frac{1}{n(n+1)} Z_{n} & n \geq 1 .\end{cases}
\end{aligned}
$$

General case (body of any shape)

Consider now a body of any shape. The following Lemma tells us how to compute the quantities we are interested in for our problem, namely the entries of the matrix $\mathbb{M}(\mathbf{c})$ and $\mathbb{N}(\mathbf{c})$.

Lemma 4.5. Let $\mathcal{S}$ be any smooth, simply connected, open bounded domain of $\mathbb{R}^{3}$ and denote $\mathcal{F}:=\mathbf{R}^{3} \backslash \overline{\mathcal{S}}$.

- Let $(\mathbf{u}, p) \in\left(W_{0}^{1}(\mathcal{F})\right)^{3} \times L^{2}(\mathcal{F})$ be any solution to the Stokes equations given by (4.5) in which only a finite number of terms are non zero (i.e. satisfying, for some $n_{0} \in \mathbf{N}, \chi_{-(n+1)}=\phi_{-(n+1)}=p_{-(n+1)}=0$ for all $\left.n>n_{0}\right)$.

- Let $\left(\mathbf{u}_{i}, p_{i}\right) \in\left(W_{0}^{1}(\mathcal{F})\right)^{3} \times L^{2}(\mathcal{F})(i=1, \ldots, 6)$ be the solution to the Stokes equations corresponding to the boundary condition

$$
\mathbf{u}_{i}(x)=\left\{\begin{array}{ll}
x \times \mathbf{e}_{i} & \text { if } i \in\{1,2,3\} \\
\mathbf{e}_{i-3} & \text { if } i \in\{4,5,6\}
\end{array} \quad \text { on } \partial \mathcal{S} .\right.
$$


Then we have,

$$
2\left(\int_{\mathcal{F}} D(\mathbf{u}): D\left(\mathbf{u}_{i}\right) \mathrm{d} x\right)_{i=1, \ldots, 6}=\left(\begin{array}{c}
-8 \pi \nabla\left(\varrho^{3} \chi_{-2}\right) \\
-4 \pi \nabla\left(\varrho^{3} p_{-2}\right)
\end{array}\right) .
$$

The idea of the proof is to turn the integral in the left hand side of (4.8) into an integral on a sphere and then to apply what has been done in the first part of this section (Spherical body case).

Proof. Let $\tilde{\mathbf{u}}_{i}$ be the rigid vector field defined by

$$
\tilde{\mathbf{u}}_{i}(x)=\left\{\begin{array}{ll}
x \times \mathbf{e}_{i} & \text { if } i \in\{1,2,3\} \\
\mathbf{e}_{i-3} & \text { if } i \in\{4,5,6\}
\end{array} .\right.
$$

Since $\mathbf{u}$ and $\mathbf{u}_{i}$ are smooth, we can integrate par parts and get:

$$
\begin{aligned}
2 \int_{\mathcal{F}} D(\mathbf{u}): D\left(\mathbf{u}_{i}\right) \mathrm{d} x & =\int_{\partial \mathcal{S}} \mathbb{T}(\mathbf{u}, p) \mathbf{u}_{i} \cdot \mathbf{n} \mathrm{d} \sigma \\
& =\int_{\partial \mathcal{S}} \mathbb{T}(\mathbf{u}, p) \tilde{\mathbf{u}}_{i} \cdot \mathbf{n} \mathrm{d} \sigma
\end{aligned}
$$

where $\mathbf{n}$ is the normal to $\partial \mathcal{S}$ oriented towards the interior of $\mathcal{S}$. Let $B(0, R) \subset \mathbf{R}^{3}$ be a ball centered at 0 of radius $R>0$ such that $\mathcal{S} \subset B(0, R)$ and denote $\mathcal{F}_{R}:=\mathcal{F} \cap B(0, R)$. Using the Green formulae and the identity

$$
D\left(\tilde{\mathbf{u}}_{i}\right):=\left(\nabla \tilde{\mathbf{u}}_{i}+\nabla \tilde{\mathbf{u}}_{i}^{*}\right) / 2=\mathbf{0}, \quad \forall i=1, \ldots, 6,
$$

we obtain

$$
\int_{\partial \mathcal{S}} \mathbb{T}(\mathbf{u}, p) \tilde{\mathbf{u}}_{i} \cdot \mathbf{n} \mathrm{d} \sigma=-\int_{\partial B(0, R)} \mathbb{T}(\mathbf{u}, p) \tilde{\mathbf{u}}_{i} \cdot \mathbf{n} \mathrm{d} \sigma,
$$

with $\mathbf{n}$ the normal to $\partial \mathcal{F}_{R}$ oriented towards the exterior of $\mathcal{F}_{R}$. Invoking the $L^{2}$ orthogonality of the spherical harmonics, we get (4.8).

\section{Application to our problem}

When the body is specialized to be the unit sphere and the boundary conditions for $\mathbf{u}$ are $\mathbf{e}_{i} \times x$ or $\mathbf{e}_{j}$ $(i, j=1,2,3)$, the entries of the vectors in (4.8) are the elements of the matrix $\mathbb{M}(c, 0)$ and we get

$$
\mathbb{M}(c, 0)=\operatorname{diag}(8 \pi \mathrm{Id}, 4 \pi \mathrm{Id}) .
$$

Similarly, if $\mathbf{u}=\mathbf{V}_{i}(i=1, \ldots, 4)$ on the surface of the body, the entries of the vectors in (4.8) turn out to be the elements of the matrix $\mathbb{N}(c, 0)$.

Let now the vector fields $\mathbf{V}_{i}$ be defined by $\mathbf{V}_{i}(\varrho, \alpha, \beta):=V_{i}(\varrho, \alpha, \beta) \mathbf{e}_{\varrho}$ for every $i \in\{1, \ldots, 4\}$ with

$$
\begin{aligned}
& V_{1}(\varrho, \alpha, \beta)=\varrho^{-(3+1)} \Re\left(Y_{3,1}\right) \\
& V_{2}(\varrho, \alpha, \beta)=\varrho^{-(3+1)} \Im\left(Y_{3,1}\right) \\
& V_{3}(\varrho, \alpha, \beta)=\varrho^{-(3+1)} \Re\left(Y_{3,2}\right) \\
& V_{4}(\varrho, \alpha, \beta)=\varrho^{-(4+1)} \Re\left(Y_{4,2}\right) .
\end{aligned}
$$

In this case, we get merely $\mathbb{N}(c, 0)=0$ and hence $\mathbf{X}_{i}(c, 0)=\mathbf{0}(i=1, \ldots, 4)$ in identity (4.3). Focusing now on the second term in the right hand side of (4.3), it remains to compute, for all $i, j=1, \ldots, 4$ and $\mathbf{c}=(c, 0)$ :

$$
\begin{aligned}
\partial_{s_{i}} \mathbf{X}_{j}(\mathbf{c})-\partial_{s_{j}} \mathbf{X}_{i}(\mathbf{c}) & =\mathbb{M}(\mathbf{c})^{-1}\left[\left(\partial_{s_{j}} \mathbb{M}(\mathbf{c}) \mathbf{X}_{i}(\mathbf{c})-\partial_{s_{i}} \mathbb{M}(\mathbf{c}) \mathbf{X}_{j}(\mathbf{c})\right)+\left(\partial_{s_{j}} \mathbb{N}(\mathbf{c}) \mathbf{f}_{i}-\partial_{s_{i}} \mathbb{N}(\mathbf{c}) \mathbf{f}_{j}\right)\right] \\
& =\mathbb{M}(\mathbf{c})^{-1}\left[\partial_{s_{j}} \mathbb{N}(\mathbf{c}) \mathbf{f}_{i}-\partial_{s_{i}} \mathbb{N}(\mathbf{c}) \mathbf{f}_{j}\right] .
\end{aligned}
$$


In particular, we need the expressions of the derivatives of the entries of the matrix $\mathbb{N}(\mathbf{c})$ with respect to $s$. We use the following Lemma:

Lemma 4.6. Let $\mathbf{V} \in C_{0}^{1}\left(\mathbf{R}^{3}\right)^{3} \cap C^{\infty}\left(\mathbf{R}^{3}\right)^{3}$ and $\mathbf{w}_{0} \in C^{\infty}(\Sigma)^{3}$ (recall that $\Sigma$ is the boundary of the unit ball $B$ and $\left.F:=\mathbf{R}^{3} \backslash \bar{B}\right)$. For every $t$ small enough, we define $\Theta_{t}=\operatorname{Id}+t \mathbf{V}, \mathcal{B}_{t}=\Theta_{t}(B), \Sigma_{t}:=\partial \mathcal{B}_{t}, \mathcal{F}_{t}=\mathbf{R}^{3} \backslash \overline{\mathcal{B}_{t}}$ and $\mathbf{w}_{t}=\mathbf{w}_{0} \circ \Theta_{t}^{-1} \in C^{\infty}\left(\Sigma_{t}\right)$.

Let also $\left(\mathbf{u}_{t}, p_{t}\right)$ and $\left(\mathbf{u}_{t}^{i}, p_{t}^{i}\right) \in\left(W_{0}^{1}\left(\mathcal{F}_{t}\right)\right)^{3} \times L^{2}\left(\mathcal{F}_{t}\right)(i=1, \ldots, 6)$ be the solutions to the Stokes problems in $\mathcal{F}_{t}$ with boundary conditions $\mathbf{u}_{t}=\mathbf{w}_{t}$ and

$$
\mathbf{u}_{t}^{i}(x)=\left\{\begin{array}{ll}
x \times \mathbf{e}_{i} & \text { if } i \in\{1,2,3\} \\
\mathbf{e}_{i-3} & \text { if } i \in\{4,5,6\}
\end{array} \quad \text { on } \Sigma_{t} .\right.
$$

Then we have

$$
\left.\frac{\mathrm{d}}{\mathrm{d} t}\left(\int_{\mathcal{F}_{t}} D\left(\mathbf{u}_{t}\right): D\left(\mathbf{u}_{t}^{i}\right) \mathrm{d} x\right)\right|_{t=0}=\int_{F} D\left(\mathbf{u}_{0}^{\prime}\right): D\left(\mathbf{u}_{t=0}^{i}\right) \mathrm{d} x
$$

where $\mathbf{u}_{0}^{\prime} \in\left(W_{0}^{1}(F)\right)^{3}$ is solution of the homogeneous Stokes problem in $F$ with the boundary condition

$$
\mathbf{u}_{0}^{\prime}=-\nabla \mathbf{u}_{t=0} \mathbf{V} \quad \text { on } \Sigma
$$

Proof. Since, for all $t$ small, the solution $\mathbf{u}_{t}$ is smooth, according to [17], Theorem 4 , the derivative of $t \mapsto \mathbf{u}_{t}$ at $t=0$ is solution of the homogeneous Stokes problem in $F$ with boundary condition (4.11) (notice that the boundary condition is merely obtained by differentiating the equation $\mathbf{u}_{t} \circ \Theta_{t}=0$ with respect to $t$ at $t=0$ ). Using the same argument as in the proof of Lemma 4.5, we have

$$
2 \int_{\mathcal{F}_{t}} D\left(\mathbf{u}_{t}\right): D\left(\mathbf{u}_{t}^{i}\right) \mathrm{d} x=-\int_{\partial B(0, R)} \mathbb{T}\left(\mathbf{u}_{t}, p_{t}\right) \tilde{\mathbf{u}}_{i} \cdot \mathbf{n} \mathrm{d} \sigma .
$$

Differentiating with respect to $t$ and invoking the linearity of $\mathbb{T}$ and the Green formulae, we get the conclusion.

This Lemma tells us that the derivatives of the entries of the matrix $\mathbb{N}(\mathbf{c})$ can be computed using the formula of Lemma 4.5 with suitable boundary conditions.

The computations are carried out using Maxima, a free software for symbolic calculation (we refer to http:// maxima. sourceforge.net/for details). The Maxima sheet we wrote can be downloaded at http://www. iecn . u-nancy.fr $/{ }^{\sim}$ munnier/Microswimmer/Maxima_sheet.wmx.

The main steps of the procedure are:

1. Computation of the functions $p_{-(n+1)}, \chi_{-(n+1)}$ and $\phi_{-(n+1)}$ arising in the formulae (4.5) for the solutions of the Stokes problem around a unit sphere with the Dirichlet boundary conditions: $\left.\mathbf{u}\right|_{\Sigma}=\mathbf{e}_{i} \times x, \mathbf{e}_{i}(i=1,2,3)$ and $\mathbf{V}_{i}(i=1, \ldots, 4)$, i.e. computation (in the form (4.5)) of the elementary Stokes flows corresponding to a rigid motion of the sphere and to elementary deformations along the vector fields $\mathbf{V}_{i}$.

2. Computation of the Jacobian matrices $\nabla \mathbf{u}$ of the solutions obtained in step 1.

3. Computation of the shape derivatives, using formula (4.11) (similar to step 1). 
These steps lead to the following results:

$$
\begin{aligned}
& \left.\partial_{s_{1}} \mathbb{N}(c, s)\right|_{s=0}=\left(\begin{array}{cccc}
0 & 0 & 0 & 0 \\
0 & 0 & -\frac{3 \sqrt{5}}{2 \frac{7}{2}^{2}} & 0 \\
0 & -\frac{3}{8} & 0 & 0 \\
0 & 0 & 0 & -\frac{\sqrt{3} \sqrt{5}}{\sqrt{2} \sqrt{7}} \\
0 & 0 & 0 & 0 \\
0 & 0 & 0 & 0
\end{array}\right),\left.\quad \partial_{s_{2}} \mathbb{N}(c, s)\right|_{s=0}=\left(\begin{array}{cccc}
0 & 0 & -\frac{3 \sqrt{5}}{2^{\frac{7}{2}}} & 0 \\
0 & 0 & 0 & 0 \\
\frac{3}{8} & 0 & 0 & 0 \\
0 & 0 & 0 & 0 \\
0 & 0 & 0 & \frac{\sqrt{3} \sqrt{5}}{\sqrt{2} \sqrt{7}} \\
0 & 0 & 0 & 0
\end{array}\right) \text {, } \\
& \left.\partial_{s_{3}} \mathbb{N}(c, s)\right|_{s=0}=\left(\begin{array}{cccc}
0 & \frac{3 \sqrt{5}}{2^{\frac{7}{2}}} & 0 & 0 \\
\frac{3 \sqrt{5}}{2^{\frac{7}{2}}} & 0 & 0 & 0 \\
0 & 0 & 0 & 0 \\
0 & 0 & 0 & 0 \\
0 & 0 & 0 & 0 \\
0 & 0 & 0 & -\frac{2 \sqrt{3}}{\sqrt{7}}
\end{array}\right),\left.\quad \partial_{s_{4}} \mathbb{N}(c, s)\right|_{s=0}=0 \text {. }
\end{aligned}
$$

One easily checks now, according to formula (4.10), that

$$
\operatorname{dim}\left(\operatorname{span}\left\{\partial_{s_{i}} \mathbb{N}(\mathbf{c}) \mathbf{f}_{j}-\partial_{s_{j}} \mathbb{N}(\mathbf{c}) \mathbf{f}_{i}, 1 \leq i<j<4\right\}\right)=6
$$

and then

$$
\operatorname{dim}\left(\operatorname{span}\left\{\mathbf{Z}_{c}^{k}(\operatorname{Id}, 0),\left[\mathbf{Z}_{c}^{i}(\operatorname{Id}, 0), \mathbf{Z}_{c}^{j}(\operatorname{Id}, 0)\right], 1 \leq k \leq 4,1 \leq i<j<4\right\}\right)=10
$$

which is the dimension of $\mathrm{SO}(3) \times \mathbf{R}^{3} \times \mathcal{S}(c)$. It entails, according to the forth point of Proposition 4.4:

Proposition 4.7. For any integer $n \geq 4$, the set of all the controllable $S S$ is an open dense subset in $\mathcal{C}(n)$.

\section{Proofs of the MAIN RESUlts}

\section{Proof of Proposition 1.6}

Let a control function $\Theta$ be given in $W^{1,1}\left([0, T], \mathcal{D}_{0}^{1}\left(\mathbf{R}^{3}\right)\right)$. With the notation of Lemma 3.2, at any time $t$ the entries of the matrix $\mathbb{M}(t)$ have the form $\Phi(\mathbf{q})$ with $\mathbf{q}:=\left(\Theta_{t}, \mathcal{W}\right), \mathcal{W}:=\left(\mathbf{W}^{1}, \mathbf{W}^{2}\right), \mathbf{W}^{j} \in\left\{\mathbf{e}_{i} \times \Theta_{t}, \mathbf{e}_{i}, i=1,2,3\right\}$ $(j=1,2)$. We deduce that

$$
t \in[0, T] \mapsto \mathbb{M}(t) \in \mathrm{M}(3)
$$

is absolutely continuous. To get the expression of the elements of the vector $\mathbf{N}(t)$ we only have to modify $\mathbf{W}^{2}$ which has to be equal to $\partial_{t} \Theta_{t}$. It entails that

$$
t \in[0, T] \mapsto \mathbf{N}(t) \in \mathbf{R}^{6}
$$

is in $L^{1}\left([0, T], \mathbf{R}^{6}\right)$. Existence of solutions is now straightforward because

$$
t \in[0, T] \mapsto \mathbb{M}(t)^{-1} \mathbf{N}(t) \in \mathbf{R}^{6}
$$

is also in $L^{1}\left([0, T], \mathbf{R}^{6}\right)$ and Carathéodory's existence theorem applies to (1.7b). Uniqueness derives from classical Grönwall's inequality.

\section{Proof of Proposition 1.7}

Let us address the stability result. With the same notation as in the statement of Proposition 1.6, denote by $\left(\boldsymbol{\Omega}^{j}, \mathbf{v}^{j}\right)^{*}$ the left hand side of identity (1.7a) when the control is $\Theta^{j}$ and $(\overline{\boldsymbol{\Omega}}, \overline{\mathbf{v}})^{*}$ when the control is $\bar{\Theta}$.

As $j \rightarrow+\infty$, it is clear that

$$
\left(\boldsymbol{\Omega}^{j}, \mathbf{v}^{j}\right)^{*} \rightarrow(\bar{\Omega}, \overline{\mathbf{v}})^{*} \text { in } L^{1}\left([0, T], \mathbf{R}^{6}\right) .
$$

Then, integrating (1.7b) between 0 and $t$ for any $0 \leq t \leq T$, we get the estimate

$$
\left\|\bar{R}(t)-R^{j}(t)\right\|_{\mathrm{M}(3)} \leq \int_{0}^{T}\left\|\bar{R}(s)-R^{j}(s)\right\|_{\mathrm{M}(3)}\|\overline{\boldsymbol{\Omega}}(s)\|_{\mathbf{R}^{3}}+\left\|\boldsymbol{\Omega}^{j}(s)-\overline{\boldsymbol{\Omega}}(s)\right\|_{\mathbf{R}^{3}} \mathrm{~d} s .
$$

Applying Grönwall's inequality, we conclude that $R^{j} \rightarrow \bar{R}$ in $C([0, T], \mathrm{M}(3))$ as $j \rightarrow+\infty$ and we use again the ODE to prove that $\dot{R}^{j} \rightarrow \dot{\bar{R}}$ in $L^{1}([0, T], \mathrm{M}(3))$. Then, it is easy to obtain the convergence of $\mathbf{r}^{j}$ to $\overline{\mathbf{r}}$ and to conclude the proof. 


\section{Proof of Theorems 1.8 and 1.9}

We shall focus on the proof of Theorem 1.8 because it will contain the proof of Theorem 1.9.

For any integer $n$, we shall use the notation

$$
d\left(c^{1}, c^{2}\right)_{\mathcal{C}(n)}:=\left\|\Theta_{\mathrm{rst}}^{1}-\Theta_{\mathrm{rst}}^{2}\right\|_{C_{0}^{1}\left(\mathbf{R}^{3}\right)^{3}}+\sum_{i=1}^{n}\left\|\mathbf{V}_{i}^{1}-\mathbf{V}_{i}^{2}\right\|_{C_{0}^{1}\left(\mathbf{R}^{3}\right)^{3}}
$$

for all $c^{1}, c^{2} \in \mathcal{D}_{0}^{1}\left(\mathbf{R}^{3}\right) \times\left(C_{0}^{1}\left(\mathbf{R}^{3}\right)^{3}\right)^{n}$ with, as usual, $c^{i}:=\left(\Theta_{\mathrm{rst}}^{i}, \mathcal{V}^{i}\right) i=1,2$ and $\mathcal{V}^{i}:=\left(\mathbf{V}_{1}^{i}, \ldots, \mathbf{V}_{n}^{i}\right)$.

Let $\varepsilon>0$ and the functions

$$
\begin{aligned}
t & \in[0, T] \mapsto \bar{\Theta}_{t} \in \mathcal{D}_{0}^{1}\left(\mathbf{R}^{3}\right) \\
\text { and } \quad t \in[0, T] & \mapsto(\bar{R}(t), \overline{\mathbf{r}}(t)) \in \mathrm{SO}(3) \times \mathbf{R}^{3}
\end{aligned}
$$

be given as in the statement of the theorem. According to Proposition C.3, we can assume that $\bar{\Theta} \in C^{\omega}\left([0, T], \mathcal{D}_{0}^{1}\left(\mathbf{R}^{3}\right)\right) \cap \mathcal{A}$ because this space is a dense subspace of $\mathcal{A}$.

Step 1 (small initial jerking of the swimmer). In this step, we prove that the swimmer is able to modify slightly its shape in order to become controllable.

Set $\bar{\Theta}^{1}:=\bar{\Theta}_{t=0}$ and $\overline{\mathbf{V}}_{1}^{1}:=\partial_{t} \bar{\Theta}_{t=0} \in C_{0}^{1}\left(\mathbf{R}^{3}\right)^{3}$. According to the self-propelled constraints (1.8), it is always possible to find three elements $\overline{\mathbf{V}}_{j}^{1}(j=2,3,4)$ in $C_{0}^{1}\left(\mathbf{R}^{3}\right)^{3}$ such that the SS $\bar{c}^{1}:=\left(\bar{\Theta}^{1}, \overline{\mathcal{V}}^{1}\right)$ belongs to $\mathcal{C}(4)$ (with $\left.\overline{\mathcal{V}}^{1}:=\left(\overline{\mathbf{V}}_{1}^{1}, \ldots, \overline{\mathbf{V}}_{4}^{1}\right)\right)$. Then, Proposition 4.7 guarantees that for any $\delta>0$ it is possible to find a controllable SS in $\mathcal{C}(4)$, denoted by $c^{1}:=\left(\Theta^{1}, \mathcal{V}^{1}\right)$ where $\mathcal{V}^{1}:=\left(\mathbf{V}_{1}^{1}, \ldots, \mathbf{V}_{4}^{1}\right)$, such that

$$
d\left(c^{1}, \bar{c}^{1}\right)_{\mathcal{C}(4)}<\delta / 2
$$

( $\delta>0$ is meant to be small an will be fixed later on). Moreover, we claim that $c^{1}$ can be chosen in such a way that there exists a smooth allowable function (i.e. satisfying (1.8))

$$
t \in[-1,0] \mapsto \Theta_{t}^{0} \in \mathcal{D}_{0}^{1}\left(\mathbf{R}^{3}\right)
$$

such that $\Theta_{t=-1}^{0}=\bar{\Theta}^{1}$ and $\Theta_{t=0}^{0}=\Theta^{1}$ (i.e. the swimmer can modify its shape from $\bar{\Theta}^{1}$ into $\Theta^{1}$ by self-deforming on time interval $[-1,0])$. Indeed, denote $\hat{c}^{1}:=\left(\hat{\Theta}^{1}, \hat{\mathcal{V}}^{1}\right) \in \mathcal{C}(4)$ a controllable SS such that $\left\|\bar{c}^{1}-\hat{c}^{1}\right\|_{\mathcal{C}(4)}$ be small. Then define

$$
\bar{\Theta}_{t}^{0}:=\bar{\Theta}^{1}+(1+t)\left(\hat{\Theta}^{1}-\bar{\Theta}^{1}\right)
$$

for every $t \in[-1,0]$. Since $\mathcal{D}_{0}^{1}\left(\mathbf{R}^{3}\right)$ is open, for $\left\|\hat{\Theta}^{1}-\bar{\Theta}^{1}\right\|_{C_{0}^{1}\left(\mathbf{R}^{3}\right)^{3}}$ small enough, $\bar{\Theta}_{t}^{0}$ will remain in $\mathcal{D}_{0}^{1}\left(\mathbf{R}^{3}\right)$ for all $t \in[-1,0]$. Then, Proposition 1.4 asserts that there exists a function

$$
Q_{0} \in A C([-1,0], \mathrm{SO}(3))
$$

and an allowable shape function

$$
\Theta_{t}^{0} \in W^{1,1}\left([-1,0], \mathcal{D}_{0}^{1}\left(\mathbf{R}^{3}\right)\right)
$$

such that $\Theta_{t}^{0} \operatorname{links} \bar{\Theta}^{1}$ (at $t=-1$ ) to some $\Theta^{1}$ (at $t=0$ ) satisfying

$$
\left.\Theta^{1}\right|_{\Sigma}=\left.Q_{0}(0) \hat{\Theta}^{1}\right|_{\Sigma} .
$$

A careful reading of the proof of Proposition 1.4 allows noticing that

$$
\left\|Q_{0}-\operatorname{Id}\right\|_{C([-1,0], \mathrm{M}(3))} \quad \text { and } \quad\left\|\Theta_{t}^{0}-\bar{\Theta}^{1}\right\|_{W^{1,1}\left([-1,0], D_{0}^{1}\left(\mathbf{R}^{3}\right)\right)}
$$

both go to 0 as $\left\|\hat{\Theta}^{1}-\bar{\Theta}^{1}\right\|_{C_{0}^{1}\left(\mathbf{R}^{3}\right)^{3}}$ goes to 0 . 
Set now $\mathbf{V}_{i}^{1}:=Q_{0}(0) \hat{\mathbf{V}}_{i}^{1}(i=1, \ldots, 4)$ and observe that the $\operatorname{SS} c^{1}=\left(\Theta^{1}, \mathcal{V}^{1}\right)$ satisfies the requirements. Furthermore, according to Proposition 1.6, $\left\|\hat{\Theta}^{1}-\bar{\Theta}^{1}\right\|_{C_{0}^{1}\left(\mathbf{R}^{3}\right)^{3}}$ can always be made small enough for the control function $\Theta_{t}^{0}$ to produce a rigid displacement

$$
t \in[-1,0] \mapsto\left(R_{0}(t), \mathbf{r}_{0}(t)\right) \in \mathrm{SO}(3) \times \mathbf{R}^{3}
$$

satisfying

$$
\sup _{t \in[-1,0]}\left(\left\|R_{0}(t)-\bar{R}(0)\right\|_{\mathrm{M}(3)}+\left\|\mathbf{r}_{0}(t)-\overline{\mathbf{r}}(0)\right\|_{\mathbf{R}^{3}}+\left\|\Theta_{t}^{0}-\bar{\Theta}^{1}\right\|_{C_{0}^{1}\left(\mathbf{R}^{3}\right)^{3}}\right)<\varepsilon / 2 .
$$

Eventually, remark that this step of initial jerking performed on the time interval $\left[t_{0}, t_{1}\right]:=[-1,0]$ can actually be carried out on a time interval arbitrarily short just by rescaling the time.

Step 2 (building a continuous piecewise $C^{1}$ control function). Since the function $\partial_{t} \bar{\Theta}$ is continuous on the compact set $[0, T]$, it is uniformly continuous. For any $\nu>0$, there exists $\delta_{\nu}>0$ such that

$$
\left\|\partial_{t} \bar{\Theta}_{t}-\partial_{t} \bar{\Theta}_{t^{\prime}}\right\|_{C_{0}^{1}\left(\mathbf{R}^{3}\right)^{3}}<\nu
$$

providing that $\left|t-t^{\prime}\right| \leq \delta_{\nu}$. We divide the time interval $[0, T]$ into

$$
0=t_{1}<t_{2}<\ldots<t_{k}=T
$$

such that $\left|t_{j+1}-t_{j}\right|<\delta_{\nu}$ for $j=1, \ldots, k-1$.

For any $t \in\left[t_{1}, t_{2}\right]$, we have the estimate:

$$
\begin{aligned}
\left\|\bar{\Theta}_{t}-\left(\Theta^{1}+\left(t-t_{1}\right) \mathbf{V}_{1}^{1}\right)\right\|_{C_{0}^{1}\left(\mathbf{R}^{3}\right)^{3}} \leq\left\|\bar{\Theta}_{t}-\left(\bar{\Theta}^{1}+\left(t-t_{1}\right) \overline{\mathbf{V}}_{1}^{1}\right)\right\|_{C_{0}^{1}\left(\mathbf{R}^{3}\right)^{3}} \\
+\left\|\bar{\Theta}^{1}-\Theta^{1}\right\|_{C_{0}^{1}\left(\mathbf{R}^{3}\right)^{3}}+\left(t-t_{1}\right)\left\|\overline{\mathbf{V}}_{1}^{1}-\mathbf{V}_{1}^{1}\right\|_{C_{0}^{1}\left(\mathbf{R}^{3}\right)^{3}} .
\end{aligned}
$$

On the one hand, we have, for all $t \in\left[t_{1}, t_{2}\right]$,

$$
\left\|\bar{\Theta}_{t}-\left(\bar{\Theta}^{1}+\left(t-t_{1}\right) \overline{\mathbf{V}}_{1}^{1}\right)\right\|_{C_{0}^{1}\left(\mathbf{R}^{3}\right)^{3}}<\nu\left|t-t_{1}\right| .
$$

On the other hand, still for $t_{1} \leq t \leq t_{2}$ and if we assume that $\delta_{\nu}<1$, we get

$$
\left\|\bar{\Theta}^{1}-\Theta^{1}\right\|_{C_{0}^{1}\left(\mathbf{R}^{3}\right)^{3}}+\left(t-t_{1}\right)\left\|\overline{\mathbf{V}}_{1}^{1}-\mathbf{V}_{1}^{1}\right\|_{C_{0}^{1}\left(\mathbf{R}^{3}\right)^{3}} \leq \delta / 2 .
$$

We denote $\bar{\Theta}^{2}:=\bar{\Theta}_{t=t_{2}}$. It is always possible to supplement $\overline{\mathbf{V}}_{1}^{2}:=\partial_{t} \bar{\Theta}_{t_{2}}$ with vector fields $\overline{\mathbf{V}}_{j}^{2}(j=2, \ldots, 4)$ in such a way that $\bar{c}^{2}:=\left(\bar{\Theta}^{2}, \overline{\mathcal{V}}^{2}\right)$ be in $\mathcal{C}(4)$ with the obvious notation $\overline{\mathcal{V}}^{2}:=\left(\overline{\mathbf{V}}_{1}^{2}, \ldots, \overline{\mathbf{V}}_{4}^{2}\right)$. We define

$$
\Theta^{2}:=\Theta^{1}+\left(t_{2}-t_{1}\right) \mathbf{V}_{1}^{1}
$$

For any $t_{1} \leq t \leq t_{2}$, Proposition 4.4 guarantees that the SS

$$
c_{t}^{1}:=\left(\Theta^{1}+\left(t-t_{1}\right) \mathbf{V}_{1}^{1}, \mathcal{V}^{1}\right)
$$

is controllable. In particular, for $t=t_{2}$, there exists an integer $k$ and a family of 10 vector fields ${ }^{3}$ in $\mathcal{E}_{k}$ (the set of all the Lie brackets of order lower or equal to $k$ ) such that the determinant of the family is nonzero. But this determinant can be thought of as an analytic function in $\mathcal{V}^{1}$. The set $\Pi^{-1}\left(\left\{\Theta^{2}\right\}\right)$ being an analytic connected submanifold of $\left(C_{0}^{1}\left(\mathbf{R}^{3}\right)^{3}\right)^{4}$ (see Cor. 2.5), the determinant is nonzero everywhere on this set but maybe in a closed subset of empty interior (for the induced topology). Therefore, it is possible to find $\mathcal{V}^{2} \in\left(C_{0}^{1}\left(\mathbf{R}^{3}\right)^{3}\right)^{4}$ such that the $\operatorname{SS} c^{2}:=\left(\Theta^{2}, \mathcal{V}^{2}\right)$ is controllable and

$$
d\left(\bar{c}^{2}, c^{2}\right)_{\mathcal{C}(4)}<\left(\delta / 2+\nu\left(t_{2}-t_{1}\right)\right)+\delta / 4 .
$$

\footnotetext{
${ }^{3} 10$ is the dimension of $\mathrm{SO}(3) \times \mathbf{R}^{3} \times \mathcal{S}\left(c_{t_{2}}^{1}\right)$.
} 
By induction, we can build $\bar{c}^{j}$ and $c^{j}(j=1,2, \ldots, k)$ such that

1. $d\left(\bar{c}^{j}, c^{j}\right)_{\mathcal{C}(4)} \leq \delta / 2+\sum_{i=2}^{k} \delta / 2^{i}+\nu\left(t_{i}-t_{i-1}\right)<\delta+\nu T$

2. every $c^{j}$ is controllable.

We choose $\delta$ and $\nu$ in such a way that

$$
\delta+\nu T<\varepsilon / 4
$$

and we define

$$
t:[0, T] \mapsto \tilde{\Theta}_{t} \in \mathcal{D}_{0}^{1}\left(\mathbf{R}^{3}\right)
$$

as continuous, piecewise affine functions by

$$
\tilde{\Theta}_{t}:=\Theta^{j}+\left(t-t_{j}\right) \mathbf{V}_{1}^{j} \quad \text { if } \quad t \in\left[t_{j}, t_{j+1}\right] \quad(j=1, \ldots, k-1) .
$$

Notice that for any $t \in[0, T]$,

$$
\left\|\bar{\Theta}_{t}-\tilde{\Theta}_{t}\right\|_{C_{0}^{1}\left(\mathbf{R}^{3}\right)^{3}}<\varepsilon / 2 .
$$

Definition 4.2 and Proposition 4.3 ensure that, on every interval $\left[t_{j}, t_{j+1}\right](j=1, \ldots, k-1)$, there exist four $C^{1}$ functions

$$
\lambda_{i}^{j}:\left[t_{j}, t_{j+1}\right] \mapsto \mathbf{R} \quad(i=1, \ldots, 4)
$$

such that the solution

$$
\left(R_{j}, \mathbf{r}_{j}, s^{j}\right):\left[t_{j}, t_{j+1}\right] \rightarrow \mathrm{SO}(3) \times \mathbf{R}^{3} \times \mathbf{R}^{4}
$$

to the ODE (4.2) with vector fields $\mathbf{Z}_{c^{j}}^{i}\left(R_{j}, s^{j}\right)$ and Cauchy data

$$
\begin{array}{ll}
R_{1}\left(t_{1}\right)=R_{0}(0), & \mathbf{r}_{1}\left(t_{1}\right)=\mathbf{r}_{0}(0), \\
R_{j}\left(t_{j}\right)=\bar{R}\left(t_{j}\right), & \mathbf{r}_{j}\left(t_{j}\right)=\overline{\mathbf{r}}\left(t_{j}\right), \quad(j=2, \ldots, k-1)
\end{array}
$$

and

$$
s^{j}\left(t_{j}\right)=0, \quad(j=1, \ldots, k-1)
$$

satisfies:

1. $\sup _{t \in\left[t_{j}, t_{j+1}\right]}\left(\left\|\bar{R}(t)-R_{j}(t)\right\|_{\mathrm{M}(3)}+\left\|\overline{\mathbf{r}}(t)-\mathbf{r}_{j}(t)\right\|_{\mathbf{R}^{3}}+\left\|\tilde{\Theta}_{t}-\Theta_{t}^{j}\right\|_{C_{0}^{1}\left(\mathbf{R}^{3}\right)^{3}}\right)<\varepsilon / 4$ with $\Theta_{t}^{j}:=\Theta^{j}+\sum_{i=1}^{4} s_{i}^{j}(t) \mathbf{V}_{i}^{j} ;$

2. $R_{j}\left(t_{j+1}\right)=\bar{R}\left(t_{j+1}\right), \mathbf{r}_{j}\left(t_{j+1}\right)=\overline{\mathbf{r}}\left(t_{j+1}\right)$ and $s^{j}\left(t_{j+1}\right)=\left(t_{j+1}-t_{j}, 0,0,0\right)^{*}$.

With these settings, the functions

$$
\begin{aligned}
t & \in[-1, T] \\
\breve{R}:[-1, T] & \mapsto \mathrm{SO}(3) \\
\text { and } \breve{\mathbf{r}}:[-1, T] & \rightarrow \mathbf{R}^{3}
\end{aligned}
$$

defined by $\breve{\Theta}_{t}:=\Theta_{t}^{j}, \breve{R}(t):=R_{j}(t)$ and $\breve{\mathbf{r}}(t):=\mathbf{r}_{j}(t)$ if $t \in\left[t_{j}, t_{j+1}\right](j=0, \ldots, k-1)$ are continuous, piecewise $C^{1}$.

Step 3 (smoothing the control function). We obtain a control function on $[0, T]$ (still denoted by $\breve{\Theta}$ ) by merely shifting/rescaling the time, from $[-1, T]$ onto $[0, T]$.

Beforehand and as already mentioned, the first time interval $\left[t_{0}, t_{1}\right]:=[-1,0]$ could have been shortened as much as necessary for the estimate

$$
\sup _{t \in[0, T]}\left(\|\bar{R}(t)-\breve{R}(t)\|_{\mathrm{M}(3)}+\|\overline{\mathbf{r}}(t)-\breve{\mathbf{r}}(t)\|_{\mathbf{R}^{3}}+\left\|\bar{\Theta}_{t}-\breve{\Theta}_{t}\right\|_{C_{0}^{1}\left(\mathbf{R}^{3}\right)^{3}}\right)<\varepsilon / 2,
$$


to be true after the shifting/rescaling process. Then, we invoke Proposition C.3 and Proposition 1.6 to conclude that there exists

$$
t \in[0, T] \mapsto \Theta_{t} \in \mathcal{D}_{0}^{1}\left(\mathbf{R}^{3}\right)
$$

analytic, satisfying (1.8) and such that

$$
\sup _{t \in[0, T]}\left(\|R(t)-\breve{R}(t)\|_{\mathrm{M}(3)}+\|\mathbf{r}(t)-\breve{\mathbf{r}}(t)\|_{\mathbf{R}^{3}}+\left\|\Theta_{t}-\breve{\Theta}_{t}\right\|_{C_{0}^{1}\left(\mathbf{R}^{3}\right)^{3}}\right)<\varepsilon / 2,
$$

where $(R, \mathbf{r}):[0, T] \mapsto \mathrm{SO}(3) \times \mathbf{R}^{3}$ is the solution to system (1.7) with initial data $(R(0), \mathbf{r}(0))=(\bar{R}(0), \overline{\mathbf{r}}(0))$ and control $\Theta$. The proof is then complete.

Proof of Theorem 1.10

Under the assumptions of the Theorem, denote by:

$$
j(t)=\int_{0}^{t} f\left(R(t), \mathbf{r}(t), \Theta_{t}, \partial_{t} \Theta_{t}\right) \mathrm{d} t,
$$

the cost functional. Since:

$$
\Theta_{t}=\Theta_{\mathrm{rst}}+\sum_{i=1}^{n} s_{i}(t) \mathbf{V}_{i} \quad \text { and } \quad \dot{s}(t)=\sum_{i=1}^{n} \lambda_{i}(t) \mathbf{f}_{i},
$$

with $\Theta_{\text {rst }}$ and $\left(\mathbf{V}_{1}, \ldots, \mathbf{V}_{n}\right)$ given, we can rather consider $f$ as a function of $(R, \mathbf{r}, s, \lambda)$ where $s=\left(s_{1}, \ldots, s_{n}\right)$ and $\lambda=\left(\lambda_{1}, \ldots, \lambda_{n}\right)$. Notice that $f$ is still continuous, convex in the fourth variable. From the dynamics in the form (4.2), we can define the extended control system:

$$
\frac{\mathrm{d}}{\mathrm{d} t}\left(\begin{array}{c}
R \\
\mathbf{r} \\
s \\
j
\end{array}\right)=\left(\begin{array}{c}
\sum_{i=1}^{n} \lambda_{i}(t) \mathbf{Z}_{c}^{i}(R, s) \\
f(R(t), \mathbf{r}(t), s(t), \lambda(t))
\end{array}\right), \quad(0<t \leq T) .
$$

By assumption $\dot{s}$ is valued in some compact, convex set of $\mathbf{R}^{n}$. It entails that $\lambda$ is also valued in a compact, convex set of $\mathbf{R}^{n}$. We deduce that, for short time, the attainable triplets $(R, \mathbf{r}, s) \in \mathrm{SO}(3) \times \mathbf{R}^{3} \times \mathbf{R}^{n}$ are included in a compact set. One can now verify that, by a slight modification of the proof, Theorem 10.3 of [1] applies to system (5.1) and leads to the conclusion of Theorem 1.10.

\section{Conclusion}

In this paper, we have proved that every $3 \mathrm{D}$ microswimmer as the ability to swim (i.e. not only moving but tracking any given trajectory). Moreover, this can be achieved by means of arbitrarily small shape changes which can be superimposed to any preassigned macro deformation. When the shape changes are expressed as a finite combination of elementary deformations (and no macro shape changes are prescribed), we have shown that only four elementary deformations are needed for the swimmer to be able to track any trajectory. In this case and when the rate of shape changes (i.e. the velocity of deformations) is valued in a compact set, an optimal control exists for a wide variety of cost functionals.

\section{Appendix A. FunCtion SPACES}

\section{Classical function spaces}

- For any open set $\Omega \subset \mathbf{R}^{3}$ (included $\left.\Omega=\mathbf{R}^{3}\right), \mathcal{D}(\Omega)$ is the space of the smooth $\left(C^{\infty}\right)$ functions, compactly supported in $\Omega$. 
- For any open set $\Omega \subset \mathbf{R}^{3}$ (included $\Omega=\mathbf{R}^{3}$ ), the set $C_{0}^{1}(\Omega)$ is the completion of $\mathcal{D}(\Omega)$ for the norm

$$
\|u\|_{C_{0}^{1}(\Omega)}:=\sup _{x \in \Omega}|u(x)|+\|\nabla u(x)\|_{\mathbf{R}^{3}} .
$$

When $\Omega=\mathbf{R}^{3}$, we get

$$
C_{0}^{1}\left(\mathbf{R}^{3}\right):=\left\{u \in C^{1}\left(\mathbf{R}^{3}\right):|u(x)| \rightarrow 0 \text { and }\|\nabla u(x)\|_{\mathbf{R}^{3}} \rightarrow 0 \text { as }\|x\|_{\mathbf{R}^{3}} \rightarrow+\infty\right\} .
$$

- The space $C_{0}^{1}\left(\mathbf{R}^{3}\right)^{3}$ is the Banach space of all of the vector fields in $\mathbf{R}^{3}$ whose every component belongs to $C_{0}^{1}\left(\mathbf{R}^{3}\right)$.

- For any Banach space $E$ and any $T>0, C^{\omega}([0, T], E)$ is the space of analytic functions on $[0, T]$, valued in $E$.

- Let now $E$ be an open subset or an embedded submanifold of an Euclidean space and $T>0$, then $A C([0, T], E)$ consists in the absolutely continuous functions from $[0, T]$ into $E$. It is endowed with the norm

$$
\|u\|_{A C([0, T], E)}:=\|u\|_{C([0, T], E)}+\int_{0}^{T}\left\|u^{\prime}(t)\right\|_{E} \mathrm{~d} t .
$$

- $C_{0}^{m}(\Omega, \mathrm{M}(k))$ ( $m$ an integer) is the Banach space of the functions of class $C^{m}$ in $\mathbf{R}^{3}$ valued in $\mathrm{M}(k)(\mathrm{M}(k)$ stands for the Banach space of the $k \times k$ matrices, $k$ a positive integer) and compactly supported in $\Omega$.

- $E_{0}^{m}(\Omega, \mathrm{M}(k))$ stands for the connected component containing the identity function of the open subset

$$
\left\{M \in C_{0}^{m}(\Omega, \mathrm{M}(k)): \operatorname{det}(M(x)) \neq 0 \forall x \in \mathbf{R}^{3}\right\} .
$$

Lemma A.1. The set

$$
\tilde{D}_{0}^{1}\left(\mathbf{R}^{3}\right):=\left\{\vartheta \in C_{0}^{1}\left(\mathbf{R}^{3}\right)^{3} \text { s.t. Id }+\vartheta \text { is a } C^{1} \text { diffeomorphism of } \mathbf{R}^{3}\right\}
$$

is open in $C_{0}^{1}\left(\mathbf{R}^{3}\right)^{3}$.

Proof. If $\vartheta \in C_{0}^{1}\left(\mathbf{R}^{3}\right)^{3}$ is such that $\|\vartheta\|_{C_{0}^{1}\left(\mathbf{R}^{3}\right)^{3}}<1$, the local inversion Theorem and a fixed point argument ensure that Id $+\vartheta$ is a $C^{1}$ diffeomorphism whence we deduce that $\tilde{D}_{0}^{1}\left(\mathbf{R}^{3}\right)$ contains the unit ball $B(0,1)$ of $C_{0}^{1}\left(\mathbf{R}^{3}\right)^{3}$.

For any $\vartheta_{0} \in \tilde{D}_{0}^{1}\left(\mathbf{R}^{3}\right)$ and any $\vartheta \in C_{0}^{1}\left(\mathbf{R}^{3}\right)^{3}$, we define:

$$
F_{\vartheta_{0}}(\vartheta):=\vartheta \circ\left(\mathrm{Id}+\vartheta_{0}\right) \in C_{0}^{1}\left(\mathbf{R}^{3}\right)^{3} .
$$

One easily verifies that $F_{\vartheta_{0}}$ is (linear) continuous from $C_{0}^{1}\left(\mathbf{R}^{3}\right)^{3}$ into $C_{0}^{1}\left(\mathbf{R}^{3}\right)^{3}$.

Observe that:

$$
\operatorname{Id}+\vartheta=\left(\operatorname{Id}+\left(\vartheta-\vartheta_{0}\right) \circ\left(\operatorname{Id}+\vartheta_{0}\right)^{-1}\right) \circ\left(\operatorname{Id}+\vartheta_{0}\right),
$$

and $\vartheta_{0}^{\dagger}:=\left(\operatorname{Id}+\vartheta_{0}\right)^{-1}-$ Id is still in $\tilde{D}_{0}^{1}\left(\mathbf{R}^{3}\right)$. Therefore, $\vartheta_{0}+F_{\vartheta_{0}^{\dagger}}^{-1}(B(0,1))$ in an open neighborhood of $\vartheta_{0}$ in $\tilde{D}_{0}^{1}\left(\mathbf{R}^{3}\right)$.

Definition A.2. We denote $D_{0}^{1}\left(\mathbf{R}^{3}\right)$ the connected component of $\tilde{D}_{0}^{1}\left(\mathbf{R}^{3}\right)$ that contains the identically zero function (recall that this set contains the unit ball of $C_{0}^{1}\left(\mathbf{R}^{3}\right)^{3}$ ) and $\mathcal{D}_{0}^{1}\left(\mathbf{R}^{3}\right):=D_{0}^{1}\left(\mathbf{R}^{3}\right)+\{\operatorname{Id}\}$ (an affine space).

Sobolev spaces

- We define the weight function $\theta(x):=\sqrt{1+|x|^{2}}\left(x \in \mathbf{R}^{3}\right)$ and the weighted Sobolev spaces:

$$
\begin{aligned}
W_{0}^{1}(\mathcal{F}) & :=\left\{u \in \mathcal{D}^{\prime}(\mathcal{F}): \theta^{-1} u \in L^{2}(\mathcal{F})\right\}, \\
\stackrel{\circ}{1}(\mathcal{F}) & :=\left\{u \in W_{0}^{1}(\mathcal{F}): \gamma_{\Sigma}(u)=0\right\},
\end{aligned}
$$

where $\gamma_{\Sigma}: W_{0}^{1}(\mathcal{F}) \mapsto H^{1 / 2}(\Sigma)$ is the classical trace operator. The dual space of $\stackrel{\circ}{W_{0}^{1}}(\mathcal{F})$ is $W_{0}^{-1}(\mathcal{F})$. 
- For any Banach space $E, W^{1,1}([0, T], E)$ is the Bochner-Sobolev spaces (see for instance [15], Sect. 7.1, p. 187) consisting in the functions

$$
u:[0, T] \mapsto E
$$

measurable and such that $u$ and $u^{\prime}$ belong to $L^{1}([0, T], E)$ (the derivative $u^{\prime}$ as to be understood in the sense of the distributions). It can be proved that $W^{1,1}([0, T], E)$ is continuously embedded in $C([0, T], E)$ and that

$$
u(t)=u(0)+\int_{0}^{t} u^{\prime}(s) \mathrm{d} s
$$

for all $t \in[0, T]$ and all $u \in W^{1,1}([0, T], E)$, where the integral is a Bochner integral (a generalization to Banach space valued functions of the Lebesgue integral). The space $W^{1,1}([0, T], E)$ is endowed with the norm

$$
\|u\|_{W^{1,1}([0, T], E)}:=\|u\|_{C([0, T], E)}+\int_{0}^{T}\left\|u^{\prime}(s)\right\|_{E} \mathrm{~d} s .
$$

Notice that, since $E$ is not assumed to be reflexive (and actually is not for our purpose), we have $W^{1,1}([0, T], E) \subset A C([0, T], E)$ with strict inclusion. We refer to [4], Appendix for details about this tricky question.

\section{Appendix B. TeChnical proofs}

Proof of Proposition 1.3.

If we denote by $\mathbf{u}_{i}(t)(i=1, \ldots, 6)$ (respectively $\mathbf{u}_{i}^{\dagger}(t)$ ) the elementary velocity fields obtained with the control function $\Theta$ (respectively $\Theta^{\dagger}$ ), it can be verified that

$$
\mathbf{u}_{i}(t, x)=Q(t)^{*} \mathbf{u}_{i}^{\dagger}(Q(t) x+\mathbf{s}(t))
$$

for every $t \in[0, T]$, every $x \in \mathcal{F}_{t}$ and every $i=1, \ldots, 6$. We deduce that

$$
\mathbb{M}(t)=\mathbb{Q}(t)^{*} \mathbb{M}^{\dagger}(t) \mathbb{Q}(t)
$$

where the elements of $\mathbb{M}(t)$ (respectively $\mathbb{M}^{\dagger}(t)$ ) have been computed with the elementary velocity fields $\mathbf{u}_{i}(t)$ (respectively $\left.\mathbf{u}_{i}^{\dagger}(t)\right)$ and $\mathbb{Q}(t) \in \mathrm{SO}(6)$ is the bloc diagonal matrix $\operatorname{diag}(Q(t), Q(t)$ ).

On the other hand, denoting respectively by

$$
\mathbf{w}_{t}(x)=\partial_{t} \Theta_{t}\left(\Theta_{t}^{-1}(x)\right) \text { and } \mathbf{w}_{t}^{\dagger}(x)=\partial_{t} \Theta_{t}^{\dagger}\left(\Theta_{t}^{\dagger-1}(x)\right)
$$

the boundary velocity of the swimmer in both cases, we get the relation:

$$
\mathbf{w}_{t}(x)+\chi(t) \times x+\boldsymbol{\zeta}(t)=Q(t)^{*} \mathbf{w}_{t}^{\dagger}(Q(t) x+\mathbf{s}(t)), \quad \forall t \in[0, T],
$$

where $\hat{\boldsymbol{\chi}}(t):=Q(t)^{*} \dot{Q}(t)$ and $\boldsymbol{\zeta}(t):=Q(t)^{*} \dot{\mathbf{s}}(t)$. With obvious notation, we deduce that

$$
\mathbf{N}(t)+\mathbb{M}(t)(\chi(t), \boldsymbol{\zeta}(t))^{*}=\mathbb{Q}(t)^{*} \mathbf{N}^{\dagger}(t) .
$$

If we set now $(\boldsymbol{\Omega}, \mathbf{v})^{*}:=-\mathbb{M}(t) \mathbf{N}(t)$ and $\left(\boldsymbol{\Omega}^{\dagger}, \mathbf{v}^{\dagger}\right)^{*}:=-\mathbb{M}^{\dagger}(t) \mathbf{N}^{\dagger}(t)$, we get the identity

$$
\left(\boldsymbol{\Omega}^{\dagger}, \mathbf{v}^{\dagger}\right)^{*}=\mathbb{Q}(t)(\boldsymbol{\Omega}-\boldsymbol{\chi}, \mathbf{v}-\boldsymbol{\zeta})^{*} .
$$

It suffices to integrate this relation, taking into account that $(Q(0), \mathbf{s}(0))=(\operatorname{Id}, \mathbf{0})$, to obtain the conclusion of the Proposition and to complete the proof. 
Proof of Proposition 1.4.

Define $\overline{\mathbf{s}}(t):=(1 / 4 \pi) \int_{\Sigma} \Theta_{t} \mathrm{~d} \sigma$ (an absolutely continuous function on $[0, T]$ ) and $\bar{\Theta}_{t}:=\Theta_{t}-\overline{\mathbf{s}}(t)$ for every $t \in[0, T]$. The matrix

$$
\mathbb{J}(t):=\int_{\Sigma}\left\|\bar{\Theta}_{t}\right\|_{\mathbf{R}^{3}}^{2} \mathrm{Id}-\bar{\Theta}_{t} \otimes \bar{\Theta}_{t} \mathrm{~d} \sigma
$$

is always definite positive since

$$
(\mathbb{J}(t) x) \cdot x=\int_{\Sigma}\left\|\bar{\Theta}_{t} \times x\right\|_{\mathbf{R}^{3}}^{2} \mathrm{~d} \sigma, \quad \forall x \in \mathbf{R}^{3} \text { and } \forall t \in[0, T] .
$$

We can then define

$$
\chi(t):=\mathbb{J}(t)^{-1} \int_{\Sigma} \partial_{t} \bar{\Theta}_{t} \times \bar{\Theta}_{t} \mathrm{~d} \sigma
$$

as a function of $L^{1}\left([0, T], \mathbf{R}^{3}\right)$. The absolutely continuous function $t \in[0, T] \mapsto Q(t) \in \mathrm{SO}(3)$ is obtained by solving the ODE:

$$
\partial_{t} Q(t)=Q(t) \hat{\chi}(t)
$$

with Cauchy data $Q(0)=$ Id (we consider here a Carathéodory solution which is unique according to Grönwall's inequality). Then, we set

$$
\mathbf{s}(t):=-Q(t) \overline{\mathbf{s}}(t) \quad \text { and } \quad \tilde{\Theta}_{t}:=Q(t) \Theta_{t}+\mathbf{s}(t) \quad \forall t \in[0, T] .
$$

The function $\tilde{\Theta}_{t}$ is in $W^{1,1}\left([0, T], C^{1}\left(\mathbf{R}^{3}\right)^{3}\right)$, satisfies (1.8) but does not take its values in $\mathcal{D}_{0}^{1}\left(\mathbf{R}^{3}\right)$ because for every $t \in[0, T]$ :

$$
\tilde{\Theta}_{t}(x)=Q(t) x+\mathbf{s}(t)+o(1) \neq x \quad \text { as } \quad\|x\|_{\mathbf{R}^{3}} \rightarrow+\infty .
$$

Let $\Omega$ and $\Omega^{\prime}$ be large balls such that

$$
\bigcup_{t \in[0, T]} \tilde{\Theta}_{t}(\bar{B}) \subset \Omega \quad \text { and } \quad \bar{\Omega} \subset \Omega^{\prime}
$$

and consider a cut-off function $\xi$ valued in $[0,1]$ and such that $\xi=1$ in $\Omega$ and $\xi=0$ in $\mathbf{R}^{3} \backslash \bar{\Omega}^{\prime}$. To complete the proof, define $\Theta_{t}^{\dagger}(x)=X\left(t, \Theta_{t=0}(x)\right)$ where $X$ is the flow associated with the Cauchy problem

$$
\begin{aligned}
& \dot{X}(t, x)=\xi(X(t, x)) \partial_{t} \tilde{\Theta}_{t} \circ \tilde{\Theta}_{t}^{-1}(X(t, x)) \quad(t>0), \\
& X(0, x)=x
\end{aligned}
$$

\section{Appendix C. Control functions smoothing}

Proposition C.3. For every $\varepsilon>0$ and every $\Theta \in \mathcal{A}$, there exists

$$
\bar{\Theta} \in C^{\omega}\left([0, T], \mathcal{D}_{0}^{1}\left(\mathbf{R}^{3}\right)\right) \cap \mathcal{A}
$$

such that

$$
\|\bar{\Theta}-\Theta\|_{W^{1,1}\left([0, T], C_{0}^{1}\left(\mathbf{R}^{3}\right)^{3}\right)}<\varepsilon
$$

and $\bar{\Theta}_{t=0}=\Theta_{t=0}$. In particular $C^{\omega}\left([0, T], \mathcal{D}_{0}^{1}\left(\mathbf{R}^{3}\right)\right) \cap \mathcal{A}$ is dense in $\mathcal{A}$.

Proof. Let $\Theta$ be in $\mathcal{A}$. Since $C^{\omega}\left([0, T], C_{0}^{1}\left(\mathbf{R}^{3}\right)^{3}\right)$ is dense in $L^{1}\left([0, T], C_{0}^{1}\left(\mathbf{R}^{3}\right)^{3}\right)$, we can always pick an element $\zeta \in C^{\omega}\left([0, T], C_{0}^{1}\left(\mathbf{R}^{3}\right)^{3}\right)$ which makes the quantity

$$
\left\|\zeta-\partial_{t} \Theta\right\|_{L^{1}\left([0, T], C_{0}^{1}\left(\mathbf{R}^{3}\right)^{3}\right)}
$$


as small as required. Define for every $t \in[0, T]$ the analytic function

$$
\tilde{\Theta}_{t}(x)=\Theta_{t=0}(x)+\int_{0}^{t} \zeta(x, s) \mathrm{d} s, \quad x \in \mathbf{R}^{3} .
$$

The quantity $\|\tilde{\Theta}-\Theta\|_{W^{1,1}\left([0, T], C_{0}^{1}\left(\mathbf{R}^{3}\right)^{3}\right)}$ can hence be made arbitrarily small. Following the lines of the proof of Proposition 1.4, we define

$$
\tilde{\mathbf{s}}(t):=(1 / 4 \pi) \int_{\Sigma} \tilde{\Theta}_{t} \mathrm{~d} \sigma, \quad \tilde{\Theta}_{t}^{\dagger}:=\tilde{\Theta}_{t}-\tilde{\mathbf{s}}(t),
$$

and

$$
\tilde{\mathbb{J}}(t):=\int_{\Sigma}\left\|\tilde{\Theta}_{t}^{\dagger}\right\|_{\mathbf{R}^{3}}^{2} \mathrm{Id}-\tilde{\Theta}_{t}^{\dagger} \otimes \tilde{\Theta}_{t}^{\dagger} \mathrm{d} \sigma
$$

(a matrix positive definite for every $t \in[0, T]$ ). The function

$$
t \in[0, T] \mapsto \tilde{\chi}(t):=\tilde{\mathbb{J}}(t)^{-1} \int_{\Sigma} \partial_{t} \tilde{\Theta}_{t}^{\dagger} \times \tilde{\Theta}_{t}^{\dagger} \mathrm{d} \sigma \in \mathbf{R}^{3},
$$

is well defined and analytic. Let us introduce as well

$$
\mathbb{J}(t):=\int_{\Sigma}\left\|\Theta_{t}\right\|_{\mathbf{R}^{3}}^{2} \mathrm{Id}-\Theta_{t} \otimes \Theta_{t} \mathrm{~d} \sigma .
$$

Observing again that the quantity $\|\tilde{\Theta}-\Theta\|_{W^{1,1}\left([0, T], C_{0}^{1}\left(\mathbf{R}^{3}\right)^{3}\right)}$ can be arbitrarily small, we draw the same conclusion for $\|\tilde{\mathbf{s}}\|_{W^{1,1}\left([0, T], \mathbf{R}^{3}\right)}$, then for

$$
\left\|\tilde{\mathbb{J}}(t)^{-1}-\mathbb{J}(t)^{-1}\right\|_{C^{0}([0, T], M(3))}
$$

and finally for $\|\tilde{\chi}\|_{L^{1}\left([0, T], \mathbf{R}^{3}\right)}$. We infer that $\|Q-\operatorname{Id}\|_{W^{1,1}([0, T], M(3))}$, where $Q$ is the solution to the Cauchy problem in $\mathrm{SO}(3)$ :

$$
\begin{aligned}
& \partial_{t} Q_{t}=Q_{t} \tilde{\boldsymbol{\chi}}(t) \\
& Q_{t=0}=\mathrm{Id}
\end{aligned}
$$

is arbitrarily small as well and that $t \in[0, T] \mapsto Q(t) \in \mathrm{SO}(3)$ is analytic.

Then we set $\Theta_{t}^{\star}=Q(t) \tilde{\Theta}_{t}^{\dagger}$. At this point, $t \in[0, T] \mapsto \Theta_{t}^{\star}$ is analytic, satisfies (1.8) but is unlikely in $\mathcal{D}_{0}^{1}\left(\mathbf{R}^{3}\right)$, because for every $t \in[0, T]$ :

$$
\Theta_{t}^{\star}(x) \rightarrow Q(t)(x-\tilde{\mathbf{s}}(t)) \neq x \text { as }\|x\|_{\mathbf{R}^{3}} \rightarrow+\infty .
$$

Notice however that for every smooth compactly supported function

$$
\xi: \mathbf{R}^{3} \rightarrow \mathbf{R}
$$

the quantity

$$
\left\|\xi\left(\Theta^{\star}-\Theta\right)\right\|_{W^{1,1}\left([0, T], C_{0}^{1}\left(\mathbf{R}^{3}\right)^{3}\right)}
$$

can be made small. Let $\Omega$ and $\Omega^{\prime}$ be large balls such that

$$
\bigcup_{t \in[0, T]} \Theta_{t}^{\star}(\bar{B}) \subset \Omega
$$

and $\bar{\Omega} \subset \Omega^{\prime}$ and define $\xi$ as a cut-off function valued in $[0,1]$ and such that $\xi=1$ in $\Omega$ and $\xi=0$ in $\mathbf{R}^{3} \backslash \bar{\Omega}^{\prime}$. To complete the proof, define $\bar{\Theta}_{t}(x)=X\left(t, \Theta_{t=0}(x)\right)$ where $X$ is the flow associated with the Cauchy problem

$$
\begin{aligned}
& \dot{X}(t, x)=\xi(X(t, x)) \partial_{t} \Theta_{t}^{\star} \circ \Theta_{t}^{\star-1}(X(t, x)), \quad(t>0), \\
& X(0, x)=x .
\end{aligned}
$$

Indeed, $\|\Theta-\bar{\Theta}\|_{W^{1,1}\left([0, T], C_{0}^{1}\left(\mathbf{R}^{3}\right)^{3}\right)}$ is small providing that $\Omega$ is big enough and $\left\|\tilde{\xi}\left(\partial_{t} \Theta_{t}^{\star}-\partial_{t} \Theta\right)\right\|_{L^{1}\left([0, T], C_{0}^{1}\left(\mathbf{R}^{3}\right)^{3}\right)}$ small enough with $\tilde{\xi}$ a cut off function equal to 1 on $\Theta_{t=0}^{-1}(\Omega)$. 


\section{Appendix D. Stokes problem And Change of VARIAbles}

\section{D.1. Well-posedness of the Stokes problem in an exterior domain}

The following results that can be found in [8]:

Theorem D.4. Let $\Sigma$ be connected an Lipschitz continuous. Then, for any $(\mathbf{f}, g, \mathbf{h}) \in\left(W_{0}^{-1}(\mathcal{F})\right)^{3} \times L^{2}(\mathcal{F}) \times$ $\left(H^{1 / 2}(\Sigma)\right)^{3}$, there exists a unique pair $(\mathbf{u}, p) \in\left(W_{0}^{1}(\mathcal{F})\right)^{3} \times L^{2}(\mathcal{F})$ such that:

$$
\begin{aligned}
-\Delta \mathbf{u}+\nabla p=\mathbf{f} & \text { in } \mathcal{F} \\
\nabla \cdot \mathbf{u}=g & \text { in } \mathcal{F}, \\
\mathbf{u}=\mathbf{h} & \text { on } \Sigma .
\end{aligned}
$$

The solution has to be understood in the weak sense, namely:

$$
\begin{aligned}
\left.\int_{\mathcal{F}} \nabla \mathbf{u}: \nabla \mathbf{v} \mathrm{d} x-\int_{\mathcal{F}} p \nabla \cdot \mathbf{v}\right) \mathrm{d} x & =\left\langle\mathbf{f}, \mathbf{v}{ }_{\left(W_{0}^{-1}\right)^{3} \times\left(\stackrel{\circ}{0}_{0}^{1}\right)^{3}}, \quad \forall \mathbf{v} \in\left(\stackrel{\circ}{\left.W_{0}^{1}(\mathcal{F})\right)^{3},}\right.\right. \\
\nabla \cdot \mathbf{u} & =g \quad \text { in } \mathcal{F}, \\
\gamma_{\Sigma}(\mathbf{u}) & =\mathbf{h} \quad \text { on } \Sigma .
\end{aligned}
$$

Besides, there exists a constant $C_{\mathcal{F}}>0$ (depending on $\mathcal{F}$ only) such that:

$$
\|\mathbf{u}\|_{\left(W_{0}^{1}(\mathcal{F})\right)^{3}}+\|p\|_{L^{2}(\mathcal{F})} \leq C_{\mathcal{F}}\left[\|\mathbf{f}\|_{\left(W_{0}^{-1}(\mathcal{F})\right)^{3}}+\|g\|_{L^{2}(\mathcal{F})}+\|\mathbf{h}\|_{\left(H^{1 / 2}(\Sigma)\right)^{3}}\right] .
$$

\section{D.2. Change of variables}

We denote, for all $\Upsilon \in \mathcal{D}_{0}^{1}\left(\bar{B}, \mathbf{R}^{3}\right), J_{\Upsilon}:=\operatorname{det}(\nabla \Upsilon)$ and we define the matrices $\mathbb{A}_{\Upsilon}:=\left(\nabla \Upsilon^{*} \nabla \Upsilon\right)^{-1} J_{\Upsilon}$ and $\mathbb{B}_{\Upsilon}:=\left(\nabla \Upsilon^{*}\right)^{-1} J_{\Upsilon}$.

Proposition D.5. If $\Sigma$ is Lipschitz continuous, for all $\Upsilon \in \mathcal{D}_{0}^{1}\left(\bar{B}, \mathbf{R}^{3}\right)$ and for all $(\mathbf{f}, g, \mathbf{h}) \in\left(W_{0}^{-1}(\mathcal{F})\right)^{3} \times$ $L^{2}(\mathcal{F}) \times\left(H^{1 / 2}(\mathcal{F})\right)^{3}$ the following problem:

$$
\begin{aligned}
\int_{\mathcal{F}} \nabla \mathbf{U}_{\Upsilon} \mathbb{A}_{\Upsilon}: \nabla \mathbf{V} \mathrm{d} x-\int_{\mathcal{F}} P_{\Upsilon} \mathbb{B}_{\Upsilon}: \nabla \mathbf{V} \mathrm{d} x & =\langle\mathbf{f}, \mathbf{V}\rangle_{\left(W_{0}^{-1}\right)^{3} \times\left(W_{0}^{1}\right)^{3}}, \quad \forall \mathbf{V} \in\left(W_{0}^{1}(\mathcal{F})\right)^{3}, \\
\mathbb{B}_{\Upsilon}: \nabla \mathbf{U}_{\Upsilon} & =g \quad \text { in } \mathcal{F}, \\
\gamma_{\Sigma}\left(\mathbf{U}_{\Upsilon}\right) & =\mathbf{h} \quad \text { on } \Sigma,
\end{aligned}
$$

admits a unique solution $\left(\mathbf{U}_{\Upsilon}, P_{\Upsilon}\right) \in\left(W_{0}^{1}(\mathcal{F})\right)^{3} \times L^{2}(\mathcal{F})$. Moreover, there exists a constant $C_{\Upsilon}(\mathcal{F})>0$ depending on $\mathcal{F}$ and $\Upsilon$ only such that:

$$
\left\|\mathbf{U}_{\Upsilon}\right\|_{\left(W_{0}^{1}(\mathcal{F})\right)^{3}}+\left\|P_{\Upsilon}\right\|_{L^{2}(\mathcal{F})} \leq C_{\Upsilon}(\mathcal{F})\left[\|\mathbf{f}\|_{\left(W_{0}^{-1}(\mathcal{F})\right)^{3}}+\|g\|_{L^{2}(\mathcal{F})}+\|\mathbf{h}\|_{\left(H^{1 / 2}(\Sigma)\right)^{3}}\right] .
$$

Proof. Let us introduce $\mathcal{F}_{\Upsilon}:=\Upsilon(\mathcal{F}), \Sigma_{\Upsilon}:=\Upsilon(\Sigma), g_{\Upsilon}:=g \circ \Upsilon^{-1} /\left(J_{\Upsilon} \circ \Upsilon^{-1}\right)$ and $\mathbf{h}_{\Upsilon}:=\mathbf{h} \circ \Upsilon^{-1}$. We denote by $\mathbf{f}_{\Upsilon}$ the distribution in $\left(W_{0}^{-1}\left(\mathcal{F}_{\Upsilon}\right)\right)^{-1}$ defined by

$$
\left\langle\mathbf{f}_{\Upsilon}, \varphi\right\rangle_{\left(W_{0}^{-1}\left(\mathcal{F}_{Y}\right)\right)^{3} \times\left(\stackrel{\circ}{W_{0}^{1}}\left(\mathcal{F}_{\Upsilon}\right)\right)^{3}}:=\langle\mathbf{f}, \varphi \circ \Upsilon\rangle_{\left(W_{0}^{-1}(\mathcal{F})\right)^{3} \times\left(\stackrel{\circ}{W}_{0}^{1}(\mathcal{F})\right)^{3}}, \quad \forall \varphi \in \stackrel{\circ}{\left.W_{0}^{1}(\mathcal{F})\right)^{3} .}
$$

This definition makes sense because there exist two constants $\alpha_{i}(\Upsilon)>0(i=1,2)$ such that

$$
\alpha_{1}(\Upsilon)\|\varphi\|_{\left(W_{0}^{1}\left(\mathcal{F}_{\Upsilon}\right)\right)^{3}} \leq\|\varphi \circ \Upsilon\|_{\left(W_{0}^{1}(\mathcal{F})\right)^{3}} \leq \alpha_{2}(\Upsilon)\|\varphi\|_{\left(W_{0}^{1}\left(\mathcal{F}_{\Upsilon}\right)\right)^{3}}
$$


for all $\varphi \in\left(W_{0}^{1}\left(\mathcal{F}_{\Upsilon}\right)\right)^{3}$. Notice that when $\mathbf{f}$ is regular enough $\left(\right.$ i.e. can be identified with a function of $\left.\left(L_{\text {loc }}^{1}(\mathcal{F})\right)^{3}\right)$ then we get merely $\mathbf{f}_{\Upsilon}:=\mathbf{f} \circ \Upsilon^{-1} /\left(J_{\Upsilon} \circ \Upsilon^{-1}\right)$. It is easy to check that, according to the properties of $\Upsilon$, the following mapping is a bicontinuous isomorphism:

$$
\begin{aligned}
R_{\Upsilon}:\left(W_{0}^{-1}(\mathcal{F})\right)^{3} \times L^{2}(\mathcal{F}) \times\left(H^{1 / 2}(\mathcal{F})\right)^{3} & \rightarrow\left(W_{0}^{-1}\left(\mathcal{F}_{\Upsilon}\right)\right)^{3} \times L^{2}\left(\mathcal{F}_{\Upsilon}\right) \times\left(H^{1 / 2}\left(\mathcal{F}_{\Upsilon}\right)\right)^{3} . \\
(\mathbf{f}, g, \mathbf{h}) & \mapsto\left(\mathbf{f}_{\Upsilon}, g_{\Upsilon}, \mathbf{h}_{\Upsilon}\right),
\end{aligned}
$$

Denote $\left(\mathbf{u}_{\Upsilon}, p_{\Upsilon}\right)=S_{\Upsilon}\left(\mathbf{f}_{\Upsilon}, g_{\Upsilon}, \mathbf{h}_{\Upsilon}\right)$ the unique solution to the Stokes problem (D.4) in $\mathcal{F}_{\Upsilon}$. The operator $S_{\Upsilon}$ is hence a bicontinuous isomorphism mapping $\left(W_{0}^{-1}(\mathcal{F})\right)^{3} \times L^{2}(\mathcal{F}) \times\left(H^{1 / 2}(\mathcal{F})\right)^{3}$ onto $\left(W_{0}^{1}\left(\mathcal{F}_{\Upsilon}\right)\right)^{3} \times L^{2}\left(\mathcal{F}_{\Upsilon}\right)$. The following operator is a bicontinuous isomorphism as well:

$$
\begin{aligned}
H_{\Upsilon}:\left(W_{0}^{1}\left(\mathcal{F}_{\Upsilon}\right)\right)^{3} \times L^{2}\left(\mathcal{F}_{\Upsilon}\right) & \rightarrow\left(W_{0}^{1}(\mathcal{F})\right)^{3} \times L^{2}(\mathcal{F}) \\
(\mathbf{v}, q) & \mapsto(\mathbf{V}, Q)=(\mathbf{v} \circ \Upsilon, q \circ \Upsilon) .
\end{aligned}
$$

The solution to problem (D.5) is provided by the operator $T_{\Upsilon}:=H_{\Upsilon} \circ S_{\Upsilon} \circ R_{\Upsilon}$ and the following diagram commutes:

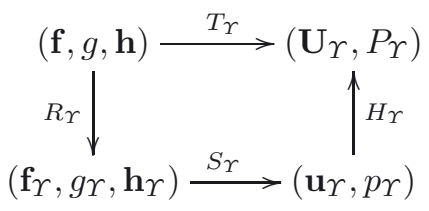

The proof is then completed.

\section{REFERENCES}

[1] A.A. Agrachev and Y.L. Sachkov, Control theory from the geometric viewpoint, vol. 87 of Encyclopaedia Math. Sci. SpringerVerlag, Berlin (2004).

[2] F. Alouges, A. DeSimone and A. Lefebvre, Optimal strokes for low Reynolds number swimmers: an example. J. Nonlinear Sci. 18 (2008) 277-302.

[3] H. Brenner. The stokes resistance of a slightly deformed sphere. Chem. Engrg. Sci. 19 (1964) 519-539.

[4] H. Brézis, Opérateurs maximaux monotones et semi-groupes de contractions dans les espaces de Hilbert. North-Holland Publishing Co., Amsterdam (1973).

[5] T. Chambrion and A. Munnier, Locomotion and control of a self-propelled shape-changing body in a fluid. J. Nonlinear Sci. 21 (2011) 325-385.

[6] T. Chambrion and A. Munnier, Generic controllability of 3d swimmers in a perfect fluid. SIAM J. Control Optim. 50 (2012) $2814-2835$.

[7] S. Childress, Mechanics of swimming and flying, vol. 2 of Cambridge Studies in Mathematical Biology, Cambridge University Press, Cambridge (1981).

[8] V. Girault and A. Sequeira, A well-posed problem for the exterior Stokes equations in two and three dimensions. Arch. Rational Mech. Anal. 114 (1991) 313-333.

[9] J. Happel and H. Brenner, Low Reynolds number hydrodynamics with special applications to particulate media. Prentice-Hall Inc., Englewood Cliffs, N.J. (1965).

[10] H. Lamb, Hydrodynamics. Cambridge Mathematical Library. 6th edition. Cambridge University Press, Cambridge, (1993).

[11] J. Lighthill, Mathematical biofluiddynamics. Society for Industrial and Applied Mathematics. Philadelphia, Pa. (1975).

[12] M.J. Lighthill, On the squirming motion of nearly spherical deformable bodies through liquids at very small Reynolds numbers. Commun. Pure Appl. Math. 5 (1952) 109-118.

[13] G. Maso, A. DeSimone and M. Morandotti, An existence and uniqueness result for the motion of self-propelled microswimmers. SIAM J. Math. Anal. 43 (2011) 1345-1368.

[14] E.M. Purcell, Life at low reynolds number. Amer. J. Phys. 45 (1977) 3-11.

[15] T. Roubíček, Nonlinear partial differential equations with applications, vol. 153 of Internat. Ser. Numer. Math. Birkhäuser Verlag, Basel (2005).

[16] A. Shapere and F. Wilczek, Geometry of self-propulsion at low Reynolds number. J. Fluid Mech. 198 (1989) $557-585$.

[17] J. Simon, Domain variation for drag in stokes flow, in vol. 159 of Control Theory of Distributed Parameter Systems and Applications, Lecture Notes in Control and Information Sciences, edited by X. Li and J. Yong. Springer Berlin/Heidelberg (1991) 28-42.

[18] G. Taylor, Analysis of the swimming of microscopic organisms. Proc. R. Soc. Lond., Ser. A 209 (1951) $447-461$.

[19] E.F. Whittlesey, Analytic functions in Banach spaces. Proc. Amer. Math. Soc. 16 (1965) 1077-1083. 